\title{
The Variational Problems for Classical N-Vector Models
}

\section{Tadeusz Balaban}

Department of Mathematics, Boston University, Boston MA 02215, USA

Received: 8 November 1994/in revised form: 15 March 1995

\begin{abstract}
We prove existence and regularity properties of solutions of the variational problems introduced in the previous paper [1] for classical lattice N-vector models. These results form a basis of our renormalization group approach to low temperature expansions for the considered models.
\end{abstract}

\section{Introduction}

In this paper we study the basic variational problems for the classical spin models introduced in [1], like the problems $(2.1),(2.2),(2.6),(2.7)$ there. We do not want to study these entirely small field cases because it is almost equally simple to consider a general case. Thus we study here the variational problems in a form appearing in a general case involving large field domains also. To formulate them we need some additional definitions. At first we have to determine a geometric setting of the problems. We consider a sequence of domains $\left\{\Omega_{j}\right\}, \Omega_{j} \subset T_{\eta}$, connected components of $\Omega_{j}$ belong to $\mathscr{D}_{j}, j=1,2, \ldots, k$ such that

$$
\Omega_{1} \supset \Omega_{2} \supset \cdots \supset \Omega_{k}, \quad\left(L^{j} \eta\right)^{-1} \operatorname{dist}\left(\Omega_{j}^{c}, \Omega_{j+1}\right)>R M,
$$

where $R$ is a positive integer which will be fixed later. Let us recall that the lattice $T_{\eta}$ and the classes $\mathscr{D}$, of localization domains have been introduced in Sect. 1 and 2 of [1]. The size of big blocks $M$ here may not be equal to the one there. In this paper we obtain conditions on $M$ connected with the variational problems, but there will be other conditions in the following papers, so we treat it as one of adjustable parameters. We define

$$
\begin{aligned}
\Lambda_{j}= & \Omega_{j}^{(j)} \backslash \Omega_{j+1}^{(j)}, \quad j=1,2, \ldots, k-1, \quad \Lambda_{k}=\Omega_{k}^{(k)}, \quad \Lambda_{0}=\Omega_{1}^{c} \cap \Omega_{1}^{\sim}, \\
& \text { where } \Omega_{j}^{(l)}=\Omega_{j} \cap T_{L^{\prime} \eta}^{(i)} \text { is the set of centers of } i \text {-blocks } \\
& \text { in } \Omega_{J}, \quad \text { hence } \Omega_{j}=B^{i}\left(\Omega_{j}^{(i)}\right), \quad \text { and } \Omega_{j}=\bigcup_{n=j}^{k} B^{n}\left(\Lambda_{n}\right) .
\end{aligned}
$$

The work has been partially supported by the NSF Grant DMS-9102639. 
Let us recall that the "tilde" operation " $\sim$ " means here that we add to $\Omega_{1}$ one layer of large cubes in the $L^{-1}$-scale "touching" the domain $\Omega_{1}$, i.e. having one of the lower dimensional edges common with some cubes of the domain. We would like to admit a case when some domains $\Omega_{j}$ are equal to $T_{\eta}$, for example $\Omega_{j}=T_{\eta}$ for $j=1, \ldots, l, l \leqq k$, and then we assume that the second condition in (1.1) is satisfied for $j \geqq l$. A sequence of domains $\left\{\Omega_{j}\right\}$ is uniquely determined by the sequence $\left\{\Lambda_{j}\right\}$, or by its union, and we define

$$
\mathbb{B}_{k}=\bigcup_{j=1}^{k} \Lambda_{j} .
$$

This set is called a generating set for the sequence $\left\{\Omega_{j}\right\}$, and in the future we will identify it with this sequence, or the sequence $\left\{\Lambda_{j}\right\}$. The idea behind these definitions is that $\Omega_{j}$ is a small field domain for the $j^{\text {th }}$ step, so we consider a composition of $j$ renormalization transformations on it. We perform a further transformation on $\Omega_{j+1}$, so we compose exactly $j$ transformations on $\Omega_{j} \backslash \Omega_{j+1}$, and the composition has the same general form as in (1.11) in [1], but the averages are taken as the averages $Q_{j}$ over $j$-blocks with centers at points of $\Lambda_{j}$, and the constant $a$ is replaced by $a_{j}\left(L^{j} \eta\right)^{-2}, a_{j}$ given by (2.11) in [1]. We do not discuss changes of the constant $\beta$ in this paper because it does not appear in the problems here. These ideas should justify the following definitions:

$$
\begin{aligned}
A\left(\mathbb{B}_{k} ; \psi, \phi ; h, a_{k}, \lambda_{k}, v_{k}\right)= & \frac{1}{2}\left\langle\psi-Q\left(\mathbb{B}_{k}\right) \phi, a\left(\mathbb{B}_{k}\right)\left(\psi-Q\left(\mathbb{B}_{k}\right) \phi\right)\right\rangle \\
& +\frac{1}{2}\left\|\partial^{\eta} \phi\right\|^{2}+\frac{\lambda_{k}}{8}\left\||\phi|^{2}-1\right\|^{2}+\frac{v_{k}}{2}\|\phi-h\|^{2},
\end{aligned}
$$

where $\phi$ is defined on $T_{\eta}$ and has values in $\mathbb{R}^{N}, \psi$ is defined on $\mathbb{B}_{k}$ and $\psi=\psi_{j}$ on $\Lambda_{j}, \psi_{j}$ is defined on $\Lambda_{j}$ and has values in $\mathbb{R}^{N}, Q\left(\mathbb{B}_{k}\right) \phi$ is defined on $\mathbb{B}_{k}$ and

$$
\begin{gathered}
\left(Q\left(\mathbb{B}_{k}\right) \phi\right)(y)=\left(Q_{j} \phi\right)(y)=\sum_{x \in B_{J}(y)} L^{-j d} \phi(x) \text { for } y \in \Lambda_{j}, \\
a\left(\mathbb{B}_{k}\right) \text { is defined on } \mathbb{B}_{k} \text { and } a\left(\mathbb{B}_{k}\right)=a_{j}\left(L^{j} \eta\right)^{-2} \text { on } \Lambda_{j}, \\
a_{j}=\frac{1-L^{-2 k}}{1-L^{-2 j}} a_{k}, \quad \text { finally } h \in \mathbb{R}^{N},|h|=1 .
\end{gathered}
$$

The norms in (1.4) are taken for appropriate scale, which means the $\eta$-scale for the last three terms on the right-hand side and the $L^{j} \eta$-scale for the part of the first term restricted to $\Lambda_{j}$. For clarity let us write explicitly the first term

$$
\begin{aligned}
\langle\psi & \left.-Q\left(\mathbb{B}_{k}\right) \phi, a\left(\mathbb{B}_{k}\right)\left(\psi-Q\left(\mathbb{B}_{k}\right) \phi\right)\right\rangle \\
& =\sum_{j=1}^{k} a_{j}\left(L^{j} \eta\right)^{-2} \sum_{y \in \Lambda_{j}}\left(L^{j} \eta\right)^{d}\left|\psi_{j}(y)-\left(Q_{j} \phi\right)(y)\right|^{2} .
\end{aligned}
$$

The coefficients $a_{k}, \lambda_{k}, v_{k}$ in this paper are arbitrary constants satisfying the assumptions of Theorem 2.2 in [1], and we denote them simply by $a, \lambda, v$, i.e. $\frac{1}{2}<a<\frac{3}{2}, \eta^{2} \lambda \geqq 1,0<v \leqq 1$. In the future we will frequently simplify the 
notations dropping the set $\mathbb{B}_{k}$ if this set is fixed. Our basic variational problem is to find

$$
\inf _{\phi \Gamma_{\Omega_{1}}} A\left(\mathbb{B}_{k} ; \psi, \phi ; h, a, \lambda, v\right),
$$

or rather more generally to find critical points of the function (1.4) in a properly defined ("small field") space of configurations $\phi$. In this problem we vary $\phi$ over the domain $\Omega_{1}$, and we keep it fixed on $\Omega_{1}^{c}$. It is convenient to reformulate the problem introducing an auxiliary variable $\alpha$, which is defined on $T_{\eta}$ and has values in $\mathbb{R}$, and the function

$$
\begin{gathered}
J\left(\mathbb{B}_{k} ; \psi, \phi, \alpha ; h, a, \lambda, v\right)=\frac{1}{2}\left\langle\psi-Q\left(\mathbb{B}_{k}\right) \phi, a\left(\mathbb{B}_{k}\right)\left(\psi-Q\left(\mathbb{B}_{k}\right) \phi\right)\right\rangle \\
+\frac{1}{2}\left\|\partial^{\eta} \phi\right\|^{2}+\frac{1}{2}\left\langle\alpha,|\phi|^{2}-1\right\rangle-\frac{1}{2 \lambda}\|\alpha\|^{2}+\frac{v}{2}\|\phi-h\|^{2} .
\end{gathered}
$$

We have

$$
A\left(\mathbb{B}_{k} ; \psi, \phi ; h, a, \lambda, v\right)=\sup _{\alpha} J\left(\mathbb{B}_{k} ; \psi, \phi, \alpha ; h, a, \lambda, v\right),
$$

so we replace the problem (1.6) by the problem

$$
\inf _{\phi \Gamma_{\Omega_{3}}} \sup _{\alpha} J\left(\mathbb{B}_{k} ; \psi, \phi, \alpha ; h, a, \lambda, v\right),
$$

and we look for critical points of the function (1.7). The corresponding variational equations for the critical points are

$$
\begin{gathered}
\left(-\Delta_{\Omega_{1}}^{D, \eta}+Q\left(\mathbb{B}_{k}\right)^{*} a\left(\mathbb{B}_{k}\right) Q\left(\mathbb{B}_{k}\right)+\alpha+v\right) \phi=Q\left(\mathbb{B}_{k}\right)^{*} a\left(\mathbb{B}_{k}\right) \psi+v h+\chi_{\partial \Omega_{1}} \Delta^{\eta} \chi_{\Omega_{1}^{c}} \phi, \\
\frac{1}{2}\left(|\phi|^{2}-1\right)-\frac{1}{\lambda} \alpha=0 .
\end{gathered}
$$

We have used the following notations here: for $\Omega \subset T_{\eta}$ we define $\partial \Omega=\{x \in \Omega$ : there exists a bond $\left\langle x, x^{\prime}\right\rangle$ of $T_{\eta}$ such that $\left.x^{\prime} \in \Omega^{c}\right\}, \chi_{\Omega}$ is a characteristic function of the set $\Omega, \Delta_{\Omega}^{D, \eta}$ is the lattice Laplace operator on $\Omega$ with Dirichlet boundary conditions, i.e. the operator defined by $\Delta_{\Omega}^{D, \eta} \phi=\chi_{\Omega} \Delta^{\eta} \chi_{\Omega} \phi$.

Let us make a few comments on the above equations. They have a very simple structure, which will be used to construct their solutions. The first is a linear equation on $\phi$, with an operator on the left-hand side which has been thoroughly investigated in the papers [2]. Under a proper smallness assumption on $\alpha$ it is a positive operator, and the equation has a unique solution equal to the corresponding inverse operator acting on the function on the right-hand side, which is expressed in terms of given elements, like $\psi, h, \phi\left\lceil\Omega_{1}^{c}\right.$. This solution may be substituted into the second equation, which is a simple local algebraic equation on $\phi, \alpha$, and we obtain an equation on $\alpha$. It has a slightly more complicated form, but eventually it may be reduced to an equation which may be solved by a contractive mapping theorem. Basically we follow this strategy, with an important exception. We construct at first a sufficiently good, almost ultra-local approximation of the solution, and then we construct the solution using the equations and following the above strategy. The objective of this is to obtain good locality properties of the solutions.

The equations (1.10) involve multiscale operators and configurations and to formulate a main theorem on their solutions we have to modify properly some of 
the definitions in [1]. These are minor modifications connected with the fact that a natural scale for the domain $\Omega_{j} \backslash \Omega_{j+1}$ is the $\xi$-scale, $\xi=L^{-j}$, not the $\eta$-scale. There are almost no modifications for the spaces $\tilde{\Psi}_{j}(\delta), \tilde{\Psi}_{j}^{c}(\delta, \varepsilon)$ given by $(3.18)$, (3.19) in [1], but these spaces are important here, so we write the complete definitions below

$$
\begin{gathered}
\tilde{\Psi}\left(\mathbb{B}_{k} ; \delta\right)=\left\{(\psi, h): \psi \text { is defined on } \mathbb{B}_{k} \cup \Lambda_{0}\right. \text { and has values } \\
\text { in } \mathbb{R}^{N}, h \in \mathbb{R}^{N},\left|\psi_{j}\left(b_{+}\right)-\psi_{j}\left(b_{-}\right)\right|<\delta \text { for } b=\left\langle b_{-}, b_{+}\right\rangle \subset \Lambda_{j}, \\
\left|\psi_{j}\left(b_{+}\right)-\psi_{j-1}(x)\right|<\delta \text { for } b \subset T^{(j)}, b_{+} \in \Lambda_{j}, b_{-} \in \Lambda_{j-1}, x \in B\left(b_{-}\right), \\
\|\left.\psi_{j}(x)\right|^{2}-1|<\delta,| v\left(1-\left(\psi_{j}(x)\right)_{0} \cdot h\right) \mid<\left(L^{j} \eta\right)^{-2} \delta^{2} \text { for } x \in \Lambda_{j}, \\
\left.\left|v\left(|h|^{2}-1\right)\right|<\delta^{2}, j=0,1, \ldots, k\right\},(\psi)_{0}=\frac{\psi}{|\psi|}, \\
\tilde{\Psi}^{c}\left(\mathbb{B}_{k} ; \delta, \varepsilon\right)=\left\{\left(\psi+\psi^{\prime}, h+h^{\prime}\right):(\psi, h) \in \tilde{\Psi}\left(\mathbb{B}_{k} ; \delta\right), \psi^{\prime}, h^{\prime}\right. \text { are }
\end{gathered}
$$

defined on $\mathbb{B}_{k} \cup \Lambda_{0}$ and have values in $\mathbb{C}^{n},\left|\psi_{j}^{\prime}\left(b_{+}\right)-\psi_{j}^{\prime}\left(b_{-}\right)\right|<\delta$ for $b \subset \Lambda_{j}$,

$$
\begin{gathered}
\left|\psi_{j}^{\prime}\left(b_{+}\right)-\psi_{j-1}^{\prime}(x)\right|<\delta \text { for } b \subset T^{(j)}, b_{+} \in \Lambda_{j}, b_{-} \in \Lambda_{j-1}, x \in B\left(b_{-}\right), \\
\left|\psi_{j}^{\prime}(x)\right|<\varepsilon+\delta,\left|\left(\psi_{j}(x)+\psi_{j}^{\prime}(x)\right)^{2}-\psi_{j}^{2}(x)\right|<\delta \\
\left|v h^{\prime}(x)\right|<\left(L^{j} \eta\right)^{-2} \frac{\delta^{2}}{\varepsilon},\left|v\left(\psi_{j}(x)\right)_{0} \cdot h^{\prime}(x)\right|<\left(L^{j} \eta\right)^{-2} \delta^{2} \text { for } x \in \Lambda_{j} \\
j=0,1,2, \ldots, k\} .
\end{gathered}
$$

These definitions means simply that the configurations $\left(\psi+\psi^{\prime}, h+h^{\prime}\right)$ restricted to $\Lambda_{j}$ belong to the spaces (3.18), (3.19) in [1], the only new element is that they are well matched for neighboring $\Lambda_{j-1}, \Lambda_{j}$. We have added the set $\Lambda_{0}$ to the generating set $\mathbb{B}_{k}$ because of the configuration $\phi\left\lceil\partial \Omega_{1}^{c}\right.$ on the right-hand side of the first equation. Notice in (1.2) that $\Lambda_{0}$ is one layer of big blocks in $\mathscr{D}_{1}$ surrounding the domain $\Omega_{1}$, and we assume that $\phi$ restricted to $\Lambda_{0}$ satisfies the conditions in (1.11). It is convenient to define.

$$
\psi_{0}=\phi\left\lceil\Lambda_{0} .\right.
$$

We want to consider Eq. (1.10) extended analytically to complex configurations $\psi, h$. The extension in $\psi$ is uniquely determined, in $h$ we take a non-unique but natural extension replacing $h$ by $h+h^{\prime}$ satisfying the conditions in (1.11), (1.12). Thus, using the simplified notation by dropping $\mathbb{B}_{k}$, we consider the system of equations

$$
\begin{gathered}
\left(-\Delta_{\Omega_{1}}^{D, \eta}+Q^{*} a Q+\alpha+v\right) \phi=Q^{*} a\left(\psi+\psi^{\prime}\right)+v\left(h+h^{\prime}\right)+\chi_{\delta \Omega_{1}} \Delta^{\eta}\left(\psi_{0}+\psi_{0}^{\prime}\right), \\
\frac{1}{2}\left(\phi^{2}-1\right)-\frac{1}{\lambda} \alpha=0
\end{gathered}
$$

where we extend $\psi_{0}+\psi_{0}^{\prime}$ onto $\Omega_{1}$ putting it equal to 0 . We can formulate now one of the main results of this paper, the following existence and uniqueness theorem: 
Proposition 1.1. Let us assume that the coefficients $a, \lambda, v$ satisfy the inequalities $\frac{1}{2}<a<\frac{3}{2}, 1 \leqq \eta^{2} \lambda \leqq+\infty, 0<v \leqq 1$. There exist positive constants $c_{0}, c_{1}, K_{1}$, $K_{1} c_{1} \leqq c_{0}$, independent of the coefficients in the above intervals, such that if $\left(\psi+\psi^{\prime}, h+h^{\prime}\right) \in \tilde{\Psi}^{c}\left(\mathbb{B}_{k} ; \delta, \varepsilon\right)$ with $\delta$, $\varepsilon$ satisfying $0<\delta \leqq \varepsilon \leqq c_{0}, \delta \leqq c_{1}, v \varepsilon^{2} \leqq$ $\delta^{2}$, then there exists a solution of the system of equations (1.14). This solution is unique in the space of all configurations $\phi, \alpha$ satisfying only the restriction $|\alpha|<$ $\left(L^{j} \eta\right)^{-2} c_{0}$ on $\Omega_{\jmath}, j=1,2, \ldots, k$. It is an analytic function of $\left(\psi+\psi^{\prime}, h+h^{\prime}\right)$ and it satisfies the following conditions on the domain $\Omega_{j}$ :

$$
\begin{gathered}
\left|\phi-Q^{*}\left(\psi+\psi^{\prime}\right)\right|<K_{1} \delta,\left|\partial^{\eta} \phi\right|<K_{1}\left(L^{j} \eta\right)^{-1} \delta, \\
\frac{1}{\left|x-x^{\prime}\right|^{1-\alpha}}\left|\left(\partial_{\mu}^{\eta} \phi\right)(x)-\left(\partial_{\mu}^{\eta} \phi\right)\left(x^{\prime}\right)\right|<K_{1} C_{\alpha}\left(L^{j} \eta\right)^{-2+\alpha} \delta, 0<\alpha \leqq 1, \\
\left|\Delta^{\eta} \phi\right|<K_{1}\left(L^{j} \eta\right)^{-2} \delta,|\alpha|<K_{1}\left(L^{J} \eta\right)^{-2} \delta,
\end{gathered}
$$

$\left|\delta \phi\left(\psi^{\prime}, h^{\prime}\right)\right|<\varepsilon+K_{1} \delta$, where $\delta \phi\left(\psi^{\prime}, h^{\prime}\right)=\phi\left(\psi+\psi^{\prime}, h+h^{\prime}\right)-\phi(\psi, h)$.

The solution corresponding to a real $(\psi, h) \in \tilde{\Psi}\left(\mathbb{B}_{k} ; \delta\right)$ is real and is a minimum of the function (1.4), hence it is a solution of the variational problem (1.6), or (1.9). It satisfies the additional conditions

$$
|\phi-h|<K_{1}\left(L^{j} \eta\right)^{-1} \frac{\delta}{\sqrt{v}},\left|v \phi \cdot Q^{*} h^{\prime}\right|<K_{1}\left(L^{j} \eta\right)^{-2} \delta^{2}
$$

on $\Omega_{j}$, for all $h^{\prime}$ satisfying the last two conditions in (1.12).

Let us make some remarks on the above proposition. The conditions (1.15), (1.16) have been formulated on $\Omega_{J}$, but the bounds improve with increasing $j$, so equivalently we may formulate them on $B^{J}\left(\Lambda_{j}\right)=\Omega_{j} \backslash \Omega_{j+1}$. The constant $C_{\alpha}$ is an absolute constant depending on $\alpha$ only, it follows from the analysis of [2(a)] that it can be taken as $O(1) \frac{1}{x^{d+1}}$. The distance $\left|x-x^{\prime}\right|$ is taken in $l^{1}$-norm, i.e. $\left|x-x^{\prime}\right|=\sum_{\mu=1}^{d}\left|x_{\mu}-x_{\mu}^{\prime}\right|$. The last condition in (1.15) means that $\delta \phi\left(\psi^{\prime}, h^{\prime}\right)$ is almost a contraction in $\psi^{\prime}$. We denote the unique solution of (1.14) by

$$
\phi\left(\mathbb{B}_{k} ; \psi+\psi^{\prime} ; h+h^{\prime}, a, \lambda, v\right), \quad \alpha\left(\mathbb{B}_{k} ; \psi+\psi^{\prime} ; h+h^{\prime}, a, \lambda, v\right) .
$$

In the future we will frequently simplify this notation dropping some of its elements, in simplest form we will write $\phi_{k}, \alpha_{k}$. It is an analytic function of $\left(\psi+\psi^{\prime}, h+h^{\prime}\right)$ defined certainly on the space $\tilde{\Psi}_{c}\left(\mathbb{B}_{k} ; c_{1}, c_{0}\right)$. It can be extended analytically also with respect to $a, \lambda, v$, but this we will study later.

Consider now the special case discussed in the first paper [1], the case where all $\Omega_{j}=T_{\eta}$. Then the space of configurations $\left(\psi+\psi^{\prime}, h+h^{\prime}\right)$ is the space $\tilde{\Psi}_{k}^{c}(\delta, \varepsilon)$, and we can take $L^{j} \eta=1$ in the bounds (1.15), (1.16). The results of Proposition 1.1, important for paper [1], can be formulated as follows:

Corollary 1.1. If $(\psi, h) \in \tilde{\Psi}_{k}(\delta), \delta \leqq c_{1}$, then the variational problem (2.2) in [1] has exactly one solution in the space of all configurations $\phi$ such that $|\alpha|<c_{0}, \alpha=\frac{\lambda}{2}\left(|\phi|^{2}-1\right)$. This solution can be analytically extended onto the 
space $\tilde{\Psi}_{k}^{c}(\delta, \varepsilon)$, if $\delta \leqq \varepsilon \leqq c_{0}, v \varepsilon^{2} \leqq \delta^{2}$, and the extension has the properties

$$
\begin{gathered}
\left(\phi_{k}\left(\psi+\psi^{\prime}, h+h^{\prime}\right), h+h^{\prime}\right): \tilde{\Psi}_{k}^{c}(\delta, \varepsilon) \rightarrow \Phi_{k}^{c}(1, \tilde{\varepsilon} ; \lambda, v), \\
\tilde{\Psi}_{k}^{c}(\delta, \varepsilon) \subset \Xi_{k}^{c}(1, \tilde{\varepsilon}),
\end{gathered}
$$

if $\varepsilon+K_{1} \delta \leqq \tilde{\varepsilon}, 2 K_{1} \delta \leqq \tilde{\varepsilon}$, or simply if $\varepsilon+2 K_{1} \delta \leqq \tilde{\varepsilon}$.

The above corollary together with the third inequality in (1.15) has been used extensively in [1].

Proposition 1.1 is the fundamental result of the paper, but we prove several other theorems; some of them play an even more important technical role in the presented renormalization group method. The rest of the paper is organized in the following way. We prove Proposition 1.1 in the next two sections, separating the case of constant configurations at first. In the fourth section we discuss generalizations of the second variational problem (2.6) in [1] and of the functions $\psi_{k}^{(j)}$.

\section{Constant Solutions and Effective Potentials}

In the next section we will construct an approximate solution of the system (1.14) by patching up some local solutions. In this section we construct and study these local, constant solutions. They are obtained by localizing the system to some sufficiently small subdomains of $T_{\eta}$, approximating the localized configuration $\left(\psi+\psi^{\prime}, h+h^{\prime}\right)$ by constant configurations, and extending the obtained systems back to the whole lattice. If we localize to a subdomain of $\Omega_{j} \backslash \Omega_{j+1}$, then we obtain a system (1.14) with $\Omega_{j}=T_{\eta}, \Omega_{j+1}=\emptyset$. It has the form

$$
\begin{gathered}
\left(-\Delta^{\eta}+a_{j}\left(L^{j} \eta\right)^{-2} Q_{j}^{*} Q_{j}+\alpha+v\right) \phi=a_{j}\left(L^{j} \eta\right)^{-2} Q_{J}^{*}\left(\psi+\psi^{\prime}\right)+v\left(h+h^{\prime}\right), \\
\frac{1}{2}\left(\phi^{2}-1\right)-\frac{1}{\lambda} \alpha=0 .
\end{gathered}
$$

It is natural to multiply it by $\left(L^{j} \eta\right)^{2}$ and to consider it on the $\xi$-lattice rather than on the $\eta$-lattice, $\xi=L^{-j}$. We get

$$
\begin{gathered}
\left(-\Delta^{\xi}+a_{j} Q_{j}^{*} Q_{j}+\left(L^{j} \eta\right)^{2} \alpha+\left(L^{j} \eta\right)^{2} v\right) \phi=a_{j} Q_{j}^{*}\left(\psi+\psi^{\prime}\right)+\left(L^{j} \eta\right)^{2} v\left(h+h^{\prime}\right), \\
\frac{1}{2}\left(\phi^{2}-1\right)-\frac{1}{\left(L^{j} \eta\right)^{2} \lambda}\left(L^{j} \eta\right)^{2} \alpha=0 .
\end{gathered}
$$

We have discussed scaling properties of $\phi, \lambda, v$ in [1], in particular see (1.10). The configurations $\phi, \psi+\psi^{\prime}, h+h^{\prime}$ are unchanged by the rescaling, and $\alpha, \lambda, v$ are rescaled to $\left(L^{j} \eta\right)^{2} \alpha,\left(L^{j} \eta\right)^{2} \lambda,\left(L^{j} \eta\right)^{2} v$. For simplicity, we denote $a_{j}$ and the rescaled quantities again by $a, \alpha, \lambda, v$ in this section, but let us write the relations for future reference,

$$
a=a_{j}, \quad \alpha=\left(L^{j} \eta\right)^{2} \alpha_{k}, \quad \lambda=\left(L^{j} \eta\right)^{2} \lambda_{k}, \quad v=\left(L^{j} \eta\right)^{2} v_{k},
$$

where we have denoted by $\alpha_{k}, \lambda_{k}, v_{k}$ the quantities in (1.14), as they have been originally denoted in (1.4). Notice that the system (2.2) is the variational system 
for the problem (2.2) in [1] for $k=j$. We consider the system (2.2) for constant configurations $\psi+\psi^{\prime}, h+h^{\prime}$, and we look for a constant solution $\phi, \alpha$. Later we will prove a uniqueness theorem which will imply that it is the only solution. We get the equations

$$
\begin{gathered}
(a+\alpha+v) \phi=a\left(\psi+\psi^{\prime}\right)+v\left(h+h^{\prime}\right), \\
\frac{1}{2}\left(\phi^{2}-1\right)-\frac{1}{\lambda} \alpha=0 .
\end{gathered}
$$

Now it is a simple system of algebraic equations for the vector $\phi$ and the scalar $\alpha$, and we can solve it calculating $\phi$ from the first linear equation, and substituting the solution into the second equation. We write this solution in the following form:

$$
\begin{aligned}
\phi= & \frac{a}{a+v+\alpha}\left(\psi+\psi^{\prime}\right)+\frac{v}{a+v+\alpha}\left(h+h^{\prime}\right) \\
= & \left(\psi+\psi^{\prime}\right)_{0}+\frac{a\left(\sqrt{\left(\psi+\psi^{\prime}\right)^{2}}-1\right)-v\left[1-\left(\psi+\psi^{\prime}\right)_{0} \cdot\left(h+h^{\prime}\right)\right]-\alpha}{a+v+\alpha}\left(\psi+\psi^{\prime}\right)_{0} \\
& +\frac{v}{a+v+\alpha}\left[I-\left(\psi+\psi^{\prime}\right)_{0} \otimes\left(\psi+\psi^{\prime}\right)_{0}\right]\left(h+h^{\prime}\right) .
\end{aligned}
$$

The reason is that we would like to use smallness of the basic invariants of the orthogonal group $O(N)$, introduced in [1] by (3.37). Let us recall these definitions:

$$
U=\sqrt{\left(\psi+\psi^{\prime}\right)^{2}}, \quad V=\left(\psi+\psi^{\prime}\right)_{0} \cdot\left(h+h^{\prime}\right), \quad W^{2}=\left(h+h^{\prime}\right)^{2}-V^{2} .
$$

The functions $U, V, W^{2}$ are complex valued functions of $\psi+\psi^{\prime}, h+h^{\prime}$, invariant with respect to simultaneous orthogonal transformations of both vectors. We consider these functions on a domain $\tilde{\Psi}_{\text {const }}^{c}(\delta, \varepsilon)$ which is naturally defined by one-point conditions in (1.11), (1.12), as domains $\tilde{\Psi}_{\text {const }}^{c}(\sigma, \varepsilon)$ in [1]. Inspecting the proofs of the inequalities (3.39), (3.41), (3.43) in [1] we see easily that the functions satisfy the inequalities with $\sigma \varepsilon$ replaced by $\delta$, assuming that $v \leqq\left(\frac{\delta}{\varepsilon}\right)^{2}$. This can be formulated as the statement

$$
\left(U, V, W^{2}\right): \tilde{\Psi}_{\text {const }}^{c}(\delta, \varepsilon) \rightarrow K(1,2 \delta) \times K\left(1,22 \frac{\delta^{2}}{v}\right) \times K\left(0,56 \frac{\delta^{4}}{v^{2} \varepsilon^{2}}\right)
$$

holding for $\varepsilon$ sufficiently small. We can simplify it using the inequality $\delta \leqq \varepsilon$, and we get

$$
\left(U, V, W^{2}\right): \tilde{\Psi}_{\text {const }}^{c}(\delta, \varepsilon) \rightarrow K(1,8 \delta) \times K\left(1, \frac{(8 \delta)^{2}}{v}\right) \times K\left(0, \frac{(8 \delta)^{2}}{v^{2}}\right) .
$$

The mapping is of course analytic. Now we substitute the solution (2.5) into the second equation in (2.4). We obtain

$$
\frac{Z-\alpha}{a+v+\alpha}+\frac{1}{2} \frac{(Z-\alpha)^{2}+v^{2} W^{2}}{(a+v+\alpha)^{2}}-\frac{1}{\lambda} \alpha=0,
$$

where $Z=a(U-1)+v(V-1)$. 
Let us replace the functions $U, V, W^{2}$ by complex variables $u, v, w^{2}$, and take $z=a(u-1)+v(v-1)$. After simple algebraic transformations we obtain the following equation:

$$
\begin{aligned}
\alpha & +\frac{\lambda+4(a+v)}{2(a+v)(\lambda+a+v)} \alpha^{2}+\frac{1}{(a+v)(\lambda+a+v)} \alpha^{3} \\
& =\frac{\lambda}{\lambda+a+v} z+\frac{\lambda}{2(a+v)(\lambda+a+v)}\left(z^{2}+v^{2} w^{2}\right) .
\end{aligned}
$$

It is a very simple equation for $\alpha$, its coefficients are bounded on the whole domain $\frac{1}{2}<a<\frac{3}{2}, 0<v \leqq 1, \lambda>0$, so for small $z, v^{2} w^{2}$ it has a unique small solution. We formulate this obvious conclusion more precisely in the following lemma.

Lemma 2.1. There exist two absolute positive constants $c_{2}, c_{3}$ such that if $\left(u, v, w^{2}\right) \in K\left(1, c_{3}\right) \times K\left(1, \frac{c_{3}^{2}}{v}\right) \times K\left(0, \frac{c_{3}^{2}}{v^{2}}\right)$, then Eq. (2.9) has a unique solution in the disc $|\alpha|<c_{2}$. This solution is an analytic function of $z, v^{2} w^{2}$ and it satisfies the bound

$$
\left|\alpha\left(z, v^{2} w^{2}\right)\right| \leqq 2\left(|z|+\left|v^{2} w^{2}\right|\right) .
$$

It is easy to find explicitly some specific constants $c_{2}, c_{3}$ analyzing carefully Eq. (2.9), but it is not important. The factor 2 in (2.10) can be replaced by any number $>1$ if $c_{3}$ is sufficiently small. Now substituting the functions $Z, W^{2}$ in place of the variables $z, w^{2}$ we obtain the function $\alpha\left(Z, v^{2} W^{2}\right)$ which is defined and analytic on any domain $\tilde{\Psi}_{\text {const }}^{c}(\delta, \varepsilon)$ such that $8 \delta \leqq c_{3}$, by $(2.8)$. This function satisfies Eq. (2.9), and substituting it into the formula (2.5) we obtain a solution of the system $(2.4)$. The solution is an analytic function on the domains $\tilde{\Psi}_{\text {const }}^{c}(\delta, \varepsilon)$, and it satisfies the bounds

$$
\begin{aligned}
& |\alpha|<2\left(3 \delta+22 \delta^{2}+56 \frac{\delta^{4}}{\varepsilon^{2}}\right)<8 \delta \text { by (2.7), assuming } 78 \delta<1, \\
& \left|\phi^{2}-1\right|=\left|\frac{2}{\lambda} \alpha\right|<\frac{16 \delta}{\lambda}, \quad \text { by the second equation in }(2.4) .
\end{aligned}
$$

Using again the proof of the inequalities (3.39), (3.41) in [1], in particular the identities (3.40), and the formula (2.5), we obtain

$$
\left|\phi-\left(\psi+\psi^{\prime}\right)_{0}\right|<O(1) \delta, \quad\left|\phi-\left(\psi+\psi^{\prime}\right)\right|<O(1) \delta
$$

with some absolute constant $O(1)$, e.g. we can take $O(1)=80$. For real solutions, i.e. the solutions for real $(\psi, h) \in \tilde{\Psi}_{\text {const }}^{c}(\delta)$, we get

$$
\begin{aligned}
|\phi-h| & \leqq\left|\phi-\psi_{0}\right|+\left|\psi_{0}-h\right|<O(1) \delta+\frac{\sqrt{3} \delta}{\sqrt{v}} \leqq O(1) \frac{\delta}{\sqrt{v}}, \\
\left|\phi \cdot h^{\prime}\right| & \leqq \frac{|a| \psi\left|+v \psi_{0} \cdot h\right|}{a+v+\alpha}\left|\psi_{0} \cdot h^{\prime}\right|+\frac{v}{a+v+\alpha}\left|\left(I-\psi_{0} \otimes \psi_{0}\right) h \| h^{\prime}\right| \\
& <6 \frac{\delta^{2}}{v}+3\left(\sqrt{3 v} \delta+\delta^{2}\right) \frac{\delta^{2}}{v \varepsilon}<12 \frac{\delta^{2}}{v}
\end{aligned}
$$


Finally, for $\delta \phi\left(\psi^{\prime}, h^{\prime}\right)=\phi\left(\psi+\psi^{\prime}, h+h^{\prime}\right)-\phi(\psi, h)$ we have

$$
\begin{gathered}
\left|\delta \phi\left(\psi^{\prime}, h^{\prime}\right)-\psi^{\prime}\right| \leqq\left|\phi\left(\psi+\psi^{\prime}, h+h^{\prime}\right)-\left(\psi+\psi^{\prime}\right)\right|+|\phi(\psi, h)-\psi|<O(1) \delta \\
\left|\delta \phi\left(\psi^{\prime}, h^{\prime}\right)\right|<\left|\psi^{\prime}\right|+O(1) \delta<\varepsilon+O(1) \delta .
\end{gathered}
$$

These inequalities give a rather crude quantitative meaning to the statement that $\delta \phi$ is almost a contraction in $\psi^{\prime}$.

We gather the obtained results in the following proposition.

Proposition 2.1. There exist positive constants $c_{2}, c_{4}, C_{1}$, independent of $a, \lambda, v$ in the intervals $\frac{1}{2}<a<\frac{3}{2}, \lambda>0,0<v \leqq 1$, such that if $0<\delta \leqq \varepsilon, v \varepsilon^{2} \leqq \delta^{2}$, $\delta \leqq c_{4}$ and $\left(\psi+\psi^{\prime}, h+h^{\prime}\right) \in \tilde{\Psi}_{\text {const }}^{c}(\delta, \varepsilon)$, then Eq. (2.4) have a unique solution in the space of all $\phi, \alpha$ satisfying only the restriction $|\alpha|<c_{2}$. The solution is an analytic function on $\tilde{\Psi}_{\text {const }}^{c}(\delta, \varepsilon)$, and it satisfies the bounds

$$
\begin{gathered}
\left|\phi-\left(\psi+\psi^{\prime}\right)_{0}\right|<C_{1} \delta,\left|\phi-\left(\psi+\psi^{\prime}\right)\right|<C_{1} \delta,|\alpha|<C_{1} \delta \\
\left|\delta \phi\left(\psi^{\prime}, h^{\prime}\right)\right|<\varepsilon+C_{1} \delta .
\end{gathered}
$$

The real solution corresponding to $(\psi, h) \in \tilde{\Psi}_{\text {const }}(\delta)$ satisfies additionally the bounds

$$
|\phi-h|<C_{1} \frac{\delta}{\sqrt{v}}, \quad\left|\phi \cdot h^{\prime}\right|<C_{1} \frac{\delta^{2}}{v}
$$

for $h^{\prime}$ satisfying the conditions in the definition (1.12).

These results serve as a basis of constructions in the next section, leading to a proof of Proposition 1.1.

In the future we will need some simple bounds for effective potentials determined by the function (1.6), i.e. the action (1.4) calculated at the solution of Eq. (1.10). In [1] we have defined the effective potentials for the localized actions $\mathscr{E}^{(j)}(y ; \psi, h)$ by the formula (2.25), taking constant configurations $\psi, h$. We can localize easily the function (1.6) and define the effective potentials in the same way. The localization will be discussed in the next paper; now we simply write the resulting formula for the effective potential corresponding to a point in $\Lambda_{J}$. We have.

$$
\left(L^{j} \eta\right)^{d-2} V_{J}(\psi, h), V_{J}(\psi, h)=\frac{a}{2}|\psi-\phi|^{2}+\frac{1}{2 \lambda} \alpha^{2}+\frac{v}{2}|\phi-h|^{2},
$$

where $\phi, \alpha$ is the solution of Eq. (2.4), and the coefficients $a, \lambda, v$ are given by (2.3). We consider here real $(\psi, h) \in \tilde{\Psi}_{\text {const }}(\delta), \delta \leqq c_{4}$, and $|h|=1$. Substituting the solution $\phi$ given by (2.5) in this case we obtain

$$
\begin{aligned}
V_{J}(\psi, h)= & \frac{1}{2} \frac{a}{(a+v+\alpha)^{2}}\left[(v+\alpha)(|\psi|-1)+v\left(1-\psi_{0} \cdot h\right)+\alpha\right]^{2} \\
& +\frac{a v}{(a+v+\alpha)^{2}} v\left(1-\psi_{0} \cdot h\right)-\frac{1}{2} \frac{a}{(a+v+\alpha)^{2}}\left[v\left(1-\psi_{0} \cdot h\right)\right]^{2}+\frac{1}{2 \lambda} \alpha^{2} \\
& +\frac{(a+\alpha)^{2}}{(a+v+\alpha)^{2}} v\left(1-\psi_{0} \cdot h\right)+\frac{a+\alpha}{(a+v+\alpha)^{2}}[a(|\psi|-1)-\alpha] v\left(1-\psi_{0} \cdot h\right) \\
& +\frac{1}{2} \frac{v}{(a+v+\alpha)^{2}}[a(|\psi|-1)-\alpha]^{2} .
\end{aligned}
$$


The function $\alpha$ is the solution of Eq. (2.9) with the right-hand side equal to

$$
\begin{aligned}
& \frac{\lambda a}{\lambda+a+v}(|\psi|-1)-\frac{\lambda a}{(a+v)(\lambda+a+v)} v\left(1-\psi_{0} \cdot h\right) \\
& +\frac{\lambda a^{2}}{2(a+v)(\lambda+a+v)}(|\psi|-1)^{2}-\frac{\lambda a}{(a+v)(\lambda+a+v)}(|\psi|-1) v\left(1-\psi_{0} \cdot h\right),
\end{aligned}
$$

hence it is an analytic function of the invariants $|\psi|-1, v\left(1-\psi_{0} \cdot h\right)$, and its expansion starts with a linear part equal to the linear part of the above expression. Thus the function $V_{j}(\psi, h)$ is also an analytic function of those invariants. Such a general statement follows from symmetry considerations as in (3.25) in [1], but the above formulas allow us to calculate explicitly lowest order terms of the expansion of the function $V_{j}$. These are a quadratic term in $|\psi|-1$ and a linear term in $v\left(1-\psi_{0} \cdot h\right)$, and this lowest order part is equal to

$$
\frac{1}{2} \frac{a(\lambda+v)}{\lambda+a+v}(|\psi|-1)^{2}+\frac{a}{a+v} v\left(1-\psi_{0} \cdot h\right) .
$$

Let us recall that the invariants are small for $(\psi, h) \in \tilde{\Psi}_{\text {const }}(\delta)$, more precisely ||$\psi|-1|<\delta,\left|v\left(1-\psi_{0} \cdot h\right)\right|<\delta^{2}$, so the lowest order part describes well the function $V_{j}$. Both terms in this part are nonnegative, so we can bound $V_{j}$ from above and from below by multiples of the above expression. To have uniform bounds we must assume additionally that $\lambda$ is not too small, e.g. $\lambda \geqq 1$. Notice that here $\lambda=\left(L^{j} \eta\right)^{2} \lambda_{k}$, but by the inductive assumption (H.8) in [1] we have $\lambda_{k}=\lambda_{0} L^{2 k}$, and we have assumed $\lambda_{0} \geqq 1$, hence $\lambda=\lambda_{0} L^{2 j} \geqq \lambda_{0} \geqq 1$. With this assumption we have the following proposition.

Proposition 2.2. There exist absolute positive constants $c_{5}, \gamma_{2}, C_{2}, c_{5} \leqq c_{4}$, such that the effective potential $V_{j}(\psi, h)$ defined by (2.17), or (2.18), satisfies the inequalities

$$
\begin{aligned}
\gamma_{2}\left[(|\psi|-1)^{2}+v\left(1-\psi_{0} \cdot h\right)\right] & \leqq V_{j}(\psi, h) \\
& \leqq C_{2}\left[(|\psi|-1)^{2}+v\left(1-\psi_{0} \cdot h\right)\right]
\end{aligned}
$$

on the domain $\tilde{\Psi}_{\text {const }}\left(c_{5}\right)$.

The above inequalities can be proved by using the representation (2.18) in quite an elementary way, although it is a bit lengthy and awkward. It can also be proved by using the lowest order part and estimating a remainder in Taylor formula for $V_{j}$. We omit the details. Let us notice that the invariant $|\psi|-1$ can be replaced by $|\psi|^{2}-1$, both are equivalent on a neighborhood of the unit sphere. We will use the above proposition, or rather the first inequality in (2.20), in the last paper only, doing estimates in large field domains.

\section{Proof of Proposition 1.1}

We prove this theorem in several steps, some of them in a slightly more general form than needed here, because we will use them in other proofs also. We start with a local approximation, and then we expand around it.

At first we construct this sufficiently good approximate local solution of Eq. (1.14). For this purpose we use constant solutions constructed and analyzed in the 
previous section. Take a configuration $\left(\psi+\psi^{\prime}, h+h^{\prime}\right) \in \tilde{\Psi^{c}}\left(\mathbb{B}_{k} ; \delta, \varepsilon\right)$ with $\delta, \varepsilon$ satisfying $0<\delta \leqq \varepsilon, v \varepsilon^{2} \leqq \delta^{2}, \delta \leqq c_{4}$. Then $\left(\psi(y)+\psi^{\prime}(y), h+h^{\prime}(y)\right) \in \tilde{\Psi^{c}}{ }_{\text {const }}(\delta, \varepsilon)$ for $y \in \mathbb{B}_{k}$, and a configuration equal to $\phi\left(\psi(y)+\psi^{\prime}(y), h+h^{\prime}(y)\right)$ on the cube $\Delta(y)$ for $y \in \Lambda_{j}$ should be such an approximation, if it is smoothed out properly. It is convenient to use here the continuous space torus instead of the lattice torus $T_{\eta}$. We denote it by $T$ and we identify $T_{\eta}$ with the set of points $T \cap\left(\eta \mathbb{Z}^{d}+\eta\left(\frac{1}{2}, \frac{1}{2}, \ldots, \frac{1}{2}\right)\right)$. The lattice $T_{L^{j} \eta}^{(j)}$ is the set of points $T \cap\left(L^{j} \eta \mathbb{Z}^{d}+\right.$ $\left.L^{j} \eta\left(\frac{1}{2}, \frac{1}{2}, \ldots, \frac{1}{2}\right)\right), \Lambda_{j} \subset T_{L^{j} \eta}^{(j)}$ and $\Delta(y)$ for $y \in \Lambda_{j}$ is the cube of the size $L^{j} \eta$ with a center at $y$. Denote by $\tilde{\Delta}(y)$ the cube of the size $L^{j+1} \eta$ with a center at $y$. The family of cubes $\left\{\tilde{\Delta}(y): y \in \mathbb{B}_{k}\right\}$ is a cover of the domain $\Omega_{1}$. We take a decomposition of unity $\left\{h_{y}\right\}$ corresponding to this cover and having the following properties:

$$
\begin{gathered}
h_{y} \in C_{0}^{2}(\tilde{\Delta}(y)), h_{y} \geqq 0,\left|\partial h_{y}\right|<2\left(L^{j} \eta\right)^{-1}, \\
\left|\partial \partial h_{y}\right|<4\left(L^{j} \eta\right)^{-2}, h_{y}=1 \text { on a neighborhood of } y, \\
\sum_{y \in \mathbb{B}_{k}} h_{y}=1 \text { on a neighborhood of } \Omega_{1} .
\end{gathered}
$$

We consider the above conditions on the continuous space, and $\partial$ denotes one of the derivatives $\hat{o}_{\mu}$. It is easy to construct such a decomposition starting with a proper function $h \in C_{0}^{2}(]-\frac{3}{2}, \frac{3}{2}[)$ and taking its products, translations and rescalings. Define a configuration $\phi_{0}$ by the formulas

$$
\begin{aligned}
\phi_{0}(x) & =\rho_{0}(x) \frac{\tilde{\phi}(x)}{\sqrt{\tilde{\phi^{2}}(x)}} \text { for } x \text { in a neighborhood of } \Omega_{1}, \\
\tilde{\phi}(x) & =\sum_{y} h_{y}(x) \phi(y), \phi(y)=\phi\left(\psi(y)+\psi^{\prime}(y), h+h^{\prime}(y)\right) \text { for } y \in \mathbb{B}_{k}, \\
\rho_{0}(x) & =\left(\sum_{y} h_{y}(x) \phi^{2}(y)\right)^{\frac{1}{2}} .
\end{aligned}
$$

We consider these functions on the continuous space, and we claim that $\phi_{0}$ restricted to points of the lattice $\Omega_{1}$ is a good approximation of a solution of Eq. (1.14). This means that it satisfies the equations with a sufficiently small error, see Lemma 3.1 below for a precise formulation. Let us define $\alpha_{0}, f_{0}$ on the lattice $\Omega_{1}$ by the equalities

$$
\begin{gathered}
\alpha_{0}=\frac{\lambda}{2}\left(\phi_{0}^{2}-1\right), \\
f_{0}=\left(-\Delta_{\Omega_{1}}^{D, \eta}+Q^{*} a Q+\alpha_{0}+v\right) \phi_{0}-Q^{*} a\left(\psi+\psi^{\prime}\right)-v\left(h+h^{\prime}\right)-\chi_{\partial \Omega_{1}} \Delta^{\eta}\left(\psi_{0}+\psi_{0}^{\prime}\right) .
\end{gathered}
$$

From the definition (3.2) we obtain $\phi_{0}^{2}=\rho_{0}^{2}$, hence

$$
\alpha_{0}=\sum_{y} h_{y} \frac{\lambda}{2}\left(\phi^{2}(y)-1\right)=\sum_{y} h_{y} \alpha(y) .
$$

We would like to prove that $\phi_{0}, \alpha_{0}$ satisfy the conditions $(1.15),(1.16)$ with $K_{1}$ replaced by some constant, and to estimate $f_{0}$. A bound for $\alpha_{0}$ is simplest to get 
using the above equality and the third inequality in (2.15). For $x \in \Delta(y), y \in \Lambda_{j}$ we have

$$
\left|\alpha_{0}(x)\right| \leqq \sum_{y^{\prime}} h_{y^{\prime}}(x)\left|\alpha\left(y^{\prime}\right)\right|<C_{1} L^{2}\left(L^{j} \eta\right)^{-2} \delta,
$$

where we have used the fact that $\alpha(y)$ in (3.4) is connected with $\alpha$ in Proposition 2.1 by the scaling (2.3). The sum in the above inequality is restricted to $y^{\prime}$ such that $\Delta\left(y^{\prime}\right)$ touches $\Delta(y)$, which may include some points $y^{\prime} \in \Lambda_{J-1}$, and then we get the additional factor $L^{2}$. To obtain remaining bounds we have to start with some preliminary ones. We would like to estimate $\phi_{0}(x)-\phi(y)$ on $\Delta(y)$. At first we consider

$$
\tilde{\phi}(x)-\phi(y)=\sum_{y^{\prime}} h_{y^{\prime}}(x)\left(\phi\left(y^{\prime}\right)-\phi(y)\right), x \in \Delta(y) .
$$

From the second inequality in (2.15) and the conditions on $\psi+\psi^{\prime}$ in the definitions (1.11), (1.12) we obtain $\left|\phi\left(y^{\prime}\right)-\phi(y)\right|<2\left(C_{1}+d\right) \delta$. Notice also that derivatives of $\phi(x)$ can be represented by the above formula with corresponding derivatives of $h_{y^{\prime}}$. Thus

$$
\begin{aligned}
|\tilde{\phi}(x)-\phi(y)| & <2\left(C_{1}+d\right) \delta,|\partial \tilde{\phi}|<4 \cdot 2^{d}\left(C_{1}+d\right)\left(L^{J} \eta\right)^{-1} \delta, \\
|\partial \partial \tilde{\phi}| & <8 \cdot 2^{d}\left(C_{1}+d\right)\left(L^{j} \eta\right)^{-2} \delta \quad \text { on } \Delta(y), y \in \Lambda_{J} .
\end{aligned}
$$

Consider next the denominator in (3.2). We have

$$
\begin{aligned}
\tilde{\phi^{2}}(x) & =\sum_{y_{1}, y_{2}} h_{y_{1}}(x) h_{y_{2}}(x) \phi\left(y_{1}\right) \cdot \phi\left(y_{2}\right) \\
& =\sum_{y_{1}, y_{2}} h_{y_{1}}(x) h_{y_{2}}(x)\left[\frac{1}{\lambda} \alpha\left(y_{1}\right)+\frac{1}{\lambda} \alpha\left(y_{2}\right)-\frac{1}{2}\left(\phi\left(y_{1}\right)-\phi\left(y_{2}\right)\right)^{2}\right]+1 \text {, hence } \\
& \left|\tilde{\phi^{2}}(x)-1\right|<2 C_{1} \frac{1}{\left(L^{j-1} \eta\right)^{2} \lambda} \delta+8\left(C_{1}+d\right)^{2} \delta^{2}<3 C_{1} \delta
\end{aligned}
$$

for $\delta$ sufficiently small, where we have used the assumption on $\lambda$ in Proposition 1.1. The number $\tilde{\phi^{2}}(x)$ is close to 1 . So $\sqrt{\tilde{\phi^{2}}(x)}$ is close to 1 , and in particular

$$
\left|\sqrt{\tilde{\phi}^{2}(x)}-1\right|<\left|\tilde{\phi^{2}}(x)-1\right|<3 C_{1} \delta .
$$

Similarly for $\rho_{0}^{2}$ we have $\left|\rho_{0}^{2}-1\right|=\left|\frac{2}{\lambda} \alpha_{0}\right|<2 C_{1} \frac{1}{\left(L^{-1} \eta\right)^{2} \lambda} \delta \leqq 2 C_{1} \delta$, hence $\rho_{0}^{2}$ and $\rho_{0}$ are close to 1 , and

$$
\left|\rho_{0}(x)-1\right|<\left|\rho_{0}^{2}(x)-1\right|<2 C_{1} \delta .
$$

It is also easy to see that the norms $|\tilde{\phi}(x)|$ are close to 1 . Indeed we can write $\tilde{\phi}(\psi+$ $\left.\psi^{\prime}, h+h^{\prime}\right)=\tilde{\phi}(\psi, h)+\delta \tilde{\phi}\left(\psi^{\prime}, h^{\prime}\right)$, as in (1.15), where the two configurations on the right-hand side are given by the same formula in (3.2) as $\tilde{\phi}$, only $\phi(y)$ is replaced by $\phi(\psi(y), h)$ and $\delta \phi\left(\psi^{\prime}(y), h^{\prime}(y)\right)$ correspondingly. From the last inequality in (2.15) we have $|\delta \tilde{\phi}|<\varepsilon+C_{1} \delta$, and from (3.8) we have $|\tilde{\phi}(\psi, h)|-1 \mid<3 C_{1} \delta$, hence

$$
|| \tilde{\phi}(x)|-1| \leqq\|\tilde{\phi}(x)|-| \tilde{\phi}(\psi, h)\|+\| \tilde{\phi}(\psi, h)|-1|<\varepsilon+4 C_{1} \delta .
$$


In particular the norms $|\tilde{\phi}(x)|$ are arbitrarily close to 1 for $\varepsilon, \delta$ sufficiently small. From (3.7)-(3.10) and the identity

$$
\phi_{0}(x)-\phi(y)=\left(\rho_{0}(x)-1\right) \frac{\tilde{\phi}(x)}{\sqrt{\tilde{\phi}^{2}(x)}}-\left(\sqrt{\tilde{\phi}^{2}(x)}-1\right) \frac{\tilde{\phi}(x)}{\sqrt{\tilde{\phi}^{2}(x)}}+(\tilde{\phi}(x)-\phi(y)),
$$

we obtain the bound

$$
\begin{gathered}
\left|\phi_{0}(x)-\phi(y)\right|<10 C_{1} \delta+2\left(C_{1}+d\right) \delta=O(1) \delta \text { on } \Delta(y), \text { hence also } \\
\left|\left(Q_{j} \phi_{0}\right)(y)-\phi(y)\right|<O(1) \delta \text { for } y \in \Lambda_{j},
\end{gathered}
$$

assuming that $\varepsilon, \delta$ are small enough. To estimate derivatives of $\phi_{0}$ we use (3.7), and we have to estimate $\partial \rho_{0}$ additionally. We have

$$
\begin{gathered}
\partial \rho_{0}=\frac{1}{2 \rho_{0}} \sum_{y} \partial h_{y} \phi^{2}(y)=\frac{1}{\rho_{0}} \sum_{y} \partial h_{y} \frac{1}{\lambda} \alpha(y), \text { hence } \\
\left|\partial \rho_{0}\right|<4 \cdot 2^{d}\left(L^{j-1} \eta\right)^{-1} C_{1} \frac{1}{\left(L^{j-1} \eta\right)^{2} \lambda} \delta \leqq 4.2^{d} L C_{1}\left(L^{j} \eta\right)^{-1} \delta \text { on } \Delta(y) .
\end{gathered}
$$

Differentiating $\phi_{0}$ we get

$$
\begin{gathered}
\partial \phi_{0}=\partial \rho_{0}\left(\tilde{\phi^{2}}\right)^{-\frac{1}{2}} \tilde{\phi}-\rho_{0}\left(\tilde{\phi^{2}}\right)^{-\frac{3}{2}}(\tilde{\phi} \cdot \partial \tilde{\phi}) \tilde{\phi}+\rho_{0}\left(\tilde{\phi^{2}}\right)^{-\frac{1}{2}} \partial \tilde{\phi}, \text { hence } \\
\left|\partial \phi_{0}\right|<O(1)\left(L^{J} \eta\right)^{-1} \delta \text { on } \Delta(y) \text { by }(3.7)-(3.12),
\end{gathered}
$$

assuming that $\varepsilon, \delta$ are sufficiently small. We have established the bounds for the continuous space derivatives but obviously the same bounds hold for lattice derivatives. We can estimate in the same way second order derivatives, and we obtain

$$
\left|\partial \partial \rho_{0}\right|,\left|\partial \partial \phi_{0}\right|<O(1)\left(L^{J} \eta\right)^{-2} \delta \quad \text { on } \Delta(y) .
$$

The constants $O(1)$ in all the above bounds are determined in a simple way by $C_{1}, L, d$ and some absolute constants, like in (3.11) or (3.12).

To estimate the function $f_{0}$ we consider at first the expression

$$
\begin{aligned}
f_{0}(x) & +\left(\Delta_{\Omega_{1}}^{D, \eta} \phi_{0}\right)(x)+\chi_{\partial \Omega_{1}}(x)\left(\Delta^{n}\left(\psi_{0}+\psi_{0}^{\prime}\right)\right)(x) \\
= & a_{j}\left(L^{j} \eta\right)^{-2}\left[\left(Q_{j} \phi_{0}\right)(y)-\left(\psi(y)+\psi^{\prime}(y)\right)\right]+\left(\alpha_{0}(x)+v\right) \phi_{0}(x)-v\left(h+h^{\prime}(y)\right) \\
= & \left(L^{j} \eta\right)^{-2}\left\{a_{j}\left[\phi(y)-\left(\psi(y)+\psi^{\prime}(y)\right)\right]+\left(\left(L^{j} \eta\right)^{2} \alpha(y)+\left(L^{j} \eta\right)^{2} v\right) \phi(y)\right. \\
& \left.-\left(L^{j} \eta\right)^{2} v\left(h+h^{\prime}(y)\right)\right\}+a_{j}\left(L^{j} \eta\right)^{-2}\left[\left(Q_{j} \phi_{0}\right)(y)-\phi(y)\right] \\
& +\left(\alpha_{0}(x)-\alpha(y)\right) \phi_{0}(x)+(\alpha(y)+v)\left(\phi_{0}(x)-\phi(y)\right) \text { on } \Delta(y), y \in \Lambda_{j} .
\end{aligned}
$$

The expression in the curly brackets is equal to 0 by the first equation in (2.4), taking into account the rescalings (2.3). The remaining expressions on the righthand side can be estimated by $O(1)\left(L^{J} \eta\right)^{-2} \delta$ using (3.11), (3.5), (2.15). Consider the second and third terms on the left-hand side of (3.15). For $x$ not in $\partial \Omega_{1}$ their 
sum is equal to $\left(\Delta^{\eta} \phi_{0}\right)(x)$ and it follows from (3.14) that it can be estimated by $O(1)\left(L^{j} \eta\right)^{-2} \delta$. If $x \in \partial \Omega_{1}$, then the sum is equal to

$$
\begin{aligned}
& \eta^{-2}\left(\Delta^{1}\left(\chi_{\Omega_{1}} \phi_{0}+\chi_{\Omega_{1}^{c}}\left(\psi+\psi^{\prime}\right)\right)\right)(x)=\eta^{-2} \sum_{x^{\prime} n . n . x}\left[\chi_{\Omega_{1}}\left(x^{\prime}\right)\left(\partial^{1} \phi_{0}\right)\left(\left\langle x, x^{\prime}\right\rangle\right)\right. \\
& \left.\quad+\chi_{\Omega_{1}^{c}}\left(x^{\prime}\right)\left(\psi_{0}^{\prime}\left(x^{\prime}\right)+\psi_{0}\left(x^{\prime}\right)-\phi_{0}(x)\right)\right] \text { (“n.n." means nearest neighbor.) }
\end{aligned}
$$

The derivatives $\left(\partial^{1} \phi_{0}\right)\left(\left\langle x, x^{\prime}\right\rangle\right)$ are bounded by $O(1) L^{-1} \delta$, from (3.13). For the second differences above we have

$$
\begin{aligned}
\phi_{0}(x)-\left(\psi_{0}\left(x^{\prime}\right)+\psi_{0}^{\prime}\left(x^{\prime}\right)\right)= & \left(\phi_{0}(x)-\phi(y)\right)+\left(\phi(y)-\left(\psi_{1}(y)+\psi_{1}^{\prime}(y)\right)\right) \\
& +\left(\psi_{1}(y)-\psi_{0}\left(x^{\prime}\right)\right)+\left(\psi_{1}^{\prime}(y)-\psi_{0}^{\prime}\left(x^{\prime}\right)\right),
\end{aligned}
$$

and using (3.11), the second inequality (2.15), and the conditions in the definitions (1.11), (1.12) we can bound them by $O(1) \delta$. Thus the sum for $x \in \partial \Omega_{1}$ can be bounded by $O(1)(L \eta)^{-2} \delta$, where $O(1)$ is again a simple function of $C_{1}, L, d$ and absolute numbers. Quite generally then the sum of the second and third terms on the left hand side of (3.15) can be bounded by $O(1)\left(L^{j} \eta\right)^{-2} \delta$ on $\Delta(y), y \in \Lambda_{j}$. Combining all the above bounds we obtain finally

$$
\left|f_{0}\right|<O(1)\left(L^{j} \eta\right)^{-2} \delta \quad \text { on } \Delta(y), y \in \Lambda_{j} .
$$

This bound gives a precise meaning to the statement that $\phi_{0}$ is a good approximation of a solution of Eq. (1.14). The function $f_{0}$ can be considered as an error of the approximation, and this error is small for $\delta$ small enough. We have obtained most of the inequalities in (1.15) for $\phi_{0}$, more precisely the second, the fourth and the fifth. The fourth holds in a stronger form, for arbitrary second order derivatives by (3.14). This implies that the first order derivatives satisfy the Lipschitz condition, i.e. the third inequality with $\alpha=0$ and $C_{\alpha}=1$. From this we can easily get the third inequality for an arbitrary $\alpha, 0 \leqq \alpha \leqq 1$, and $C_{\alpha}=1$. The first and the last inequalities follow from (3.11) and (2.15). Consider now a real $\phi_{0}$, i.e. we take $\phi_{0}(\psi, h)$. From (3.8), (3.9), (3.11) and (2.16) we have

$$
\begin{gathered}
\left|\phi_{0}-h\right| \leqq\left|\rho_{1}-1\right|+|| \tilde{\phi}|-1|+|\tilde{\phi}-h|<5 C_{1} \delta+\sum_{y^{\prime}} h_{y^{\prime}}\left|\phi\left(y^{\prime}\right)-h\right| \\
<5 C_{1} \delta+C_{1} L\left(L^{j} \eta\right)^{-1} \frac{\delta}{\sqrt{v}} \leqq 2 L C_{1}\left(L^{j} \eta\right)^{-1} \frac{\delta}{\sqrt{v}} \text { on } \Delta(y), \text { and } \\
\left|\phi_{0}(x) \cdot h^{\prime}(y)\right| \leqq\left|\left(\phi_{0}(x)-\phi(y)\right) \cdot h^{\prime}(y)\right|+\left|\phi(y) \cdot h^{\prime}(y)\right| \\
<O(1) \delta \cdot\left(L^{j} \eta\right)^{-2} \frac{\delta^{2}}{v \varepsilon}+C_{1}\left(L^{j} \eta\right)^{-2} \frac{\delta^{2}}{v} \leqq O(1)\left(L^{j} \eta\right)^{-2} \frac{\delta^{2}}{v}
\end{gathered}
$$

Thus we have proved the following lemma.

Lemma 3.1. The functions $\phi_{0}, \alpha_{0}, f_{0}$ defined by (3.2), (3.3) are analytic functions of $\left(\psi+\psi^{\prime}, h+h^{\prime}\right)$ on any space $\tilde{\Psi^{c}}\left(\mathbb{B}_{k} ; \delta, \varepsilon\right)$ and $\delta, \varepsilon$ satisfying the assumptions of Propositon 2.1, $\varepsilon$ sufficiently small. There exists a constant $C_{3}$ depending on $C_{1}, L$ and $d$ only, and such that $\phi_{0}, \alpha_{0}$ satisfy the conditions (1.15), (1.16) of Proposition 1.1 with $K_{1}$ replaced by $C_{3}$. The function $f_{0}$ satisfies the condition

$$
\left|f_{0}\right|<C_{3}\left(L^{j} \eta\right)^{-2} \delta \quad \text { on } \Delta(y), y \in \Lambda_{j} .
$$


We have finished the first step of the proof of Proposition 1.1, the construction of the approximate solution $\phi_{0}, \alpha_{0}$. Let us notice that these functions have one additional very important property, they are almost local functions of $\left(\psi+\psi^{\prime}\right.$, $\left.h+h^{\prime}\right)$. More precisely values $\phi_{0}(x), \alpha_{0}(x)$ for $x \in \Delta(y)$ depend on the configurations $\left(\psi+\psi^{\prime}, h+h^{\prime}\right)$ restricted to the union of all $\Delta\left(y^{\prime}\right)$ touching $\Delta(y)$, which we denote by $\tilde{\Delta}(y)$. This property is crucial for constructions of various localization expansions in the future.

In the inequalities above we have been using systematically the scaling factors given by powers of $L^{j} \eta$. It is convenient at this point to introduce appropriate definitions and notations. We define

$$
\begin{gathered}
|f|_{\sigma}=\max _{j} \sup _{x \in \Omega_{j} \backslash \Omega_{j+1}}\left(L^{j} \eta\right)^{\sigma}|f(x)|, \\
|f|_{(\alpha), \sigma}=\max _{j} \sup _{y \in \Lambda_{j}} \sup _{x \in \Delta(y)} \sup _{x^{\prime} \in \tilde{\Delta}(y)} \frac{\left(L^{j} \eta\right)^{\sigma}}{\left|x-x^{\prime}\right|^{1-\alpha}}\left|f(x)-f\left(x^{\prime}\right)\right| .
\end{gathered}
$$

We assume here that the generating set $\mathbb{B}_{k}$ is given and fixed. If there are more generating sets, then we have to introduce an additional subscript indicating which set we use in the above definitions. The above norms depend on powers of the scaling factor $L^{j} \eta$, and in all statements below we keep track of scaling properties by formulating them in terms of these norms.

A next step is to write functions $\phi, \alpha$ as perturbations of $\phi_{0}, \alpha_{0}$, and to write equations for the perturbations. We write

$$
\phi=\phi_{0}+\delta \phi, \quad \alpha=\alpha_{0}+\delta \alpha,
$$

and we take $\psi+\psi^{\prime}+\delta \psi$ in Eq. (1.14), to cover some other applications discussed later. The configurations $\delta \psi$ are defined on $\mathbb{B}_{k}$, have values in $\mathbb{C}^{N}$, and we assume that they are small. Substituting the above functions into Eq. (1.14), and using Eq. (3.3) we obtain the following system:

$$
\begin{gathered}
\left(-\Delta_{\Omega_{1}}^{D, \eta}+Q^{*} a Q+v+\alpha_{0}+\delta \alpha\right) \delta \phi+\delta \alpha \phi_{0}+f_{0}=Q^{*} a \delta \psi, \\
\frac{1}{2}\left(2 \phi_{0} \cdot \delta \phi+(\delta \phi)^{2}\right)-\frac{1}{\lambda} \delta \alpha=0,
\end{gathered}
$$

where we have included the configuration $\delta \psi_{0}$ into the definition (3.3) of the function $f_{0}$, together with $\psi_{0}+\psi_{0}^{\prime}$. We slightly generalize again the above system writing $f_{1}$ instead of $-f_{0}$ and considering $f_{1}$ as another independent functional variable. We replace also 0 by $\frac{1}{\lambda} \alpha_{1}$ on the right-hand side of the second equation. Thus we consider the system

$$
\begin{gathered}
\left(-\Delta_{\Omega_{1}}^{D, \eta}+Q^{*} a Q+v+\alpha_{0}+\delta \alpha\right) \delta \phi=Q^{*} a \delta \psi-\delta \alpha \phi_{0}+f_{1}, \\
\frac{1}{2}\left(2 \phi_{0} \cdot \delta \phi+(\delta \phi)^{2}\right)-\frac{1}{\lambda} \delta \alpha=\frac{1}{\lambda} \alpha_{1} .
\end{gathered}
$$

We look for a solution $\delta \phi, \delta \alpha$ of this system. Such a solution depends on configurations $\delta \psi, \phi_{0}, \alpha_{0}, f_{1}, \alpha_{1}$, and we now assume that they are all independent complex functional variables. Let us define a space of these configurations determined by 
positive constants $\delta_{0}, \delta_{1}$ by the following conditions:

$$
\begin{gathered}
|\delta \psi|<\delta_{1}, \quad \frac{1}{2}<\left|\phi_{0}\right|<\frac{3}{2}, \quad\left|\phi_{0}^{2}-1\right|<\delta_{0}, \quad\left|\partial^{\eta} \phi_{0}\right|_{1}<\delta_{0}, \\
\left|\Delta^{\eta} \phi_{0}\right|_{2}<\delta_{0}, \quad\left|\alpha_{0}\right|_{2}<\delta_{0}, \quad\left|f_{1}\right|_{2}<\delta_{1}, \quad\left|\alpha_{1}\right|_{2}<\delta_{1} .
\end{gathered}
$$

The configurations $\phi_{0}, \alpha_{0}, f_{1}=-f_{0}$ constructed above belong to the space with $\delta_{0}=\delta_{1}=C_{3} \delta$ for $\varepsilon$ sufficiently small. In the rest of this section we study mainly the system of equations (3.20). Our basic goal is to prove an existence and uniqueness theorem for solutions of the system. We obtain such results, under appropriate assumptions, and they are formulated in Proposition 3.1 below. Let us start with an analysis of the first equation in the system (3.20). It is linear in $\delta \phi$, and the corresponding linear operator with $\alpha_{0}+\delta \alpha=0$ has been thoroughly investigated in the paper [2(b)], Sect. B, in particular see Proposition 2.1. It is a positive operator and its inverse is bounded in proper scaled norms (3.17), see (2.57) in [2(b)]. Therefore the inverse to the linear operator in (3.20) exists also for $\alpha=\alpha_{0}+\delta \alpha$ sufficiently small, and it can be constructed by the usual Neumann series expansion

$$
\begin{aligned}
G(\alpha) & =\left(-\Delta_{\Omega_{1}}^{D, \eta}+Q^{*} a Q+v+\alpha\right)^{-1}=G-G \alpha G(\alpha) \\
& =\sum_{n=0}^{\infty} G(-\alpha G)^{n}, \text { where } G=G(0) .
\end{aligned}
$$

Using the bounds (2.57) in [2(b)] we can prove easily that there exists a constant $\gamma_{1}$ such that the series is convergent in all the norms occurring in the bounds, if $|\alpha|_{2}<\gamma_{1}$. Then the operator $G(\alpha)$ is an analytic function of $\alpha$ on this domain, and it satisfies the bounds (2.57) in [2(b)] with the constant multiplied by 2 . We denote this constant by $B_{0}$ here. Later on we will need even more detailed and precise bounds, so we postpone explanations of what is written above to a proper place. Assuming smallness of $\alpha_{0}$ and $\delta \alpha$, for example assuming $\delta_{0} \leqq \frac{1}{2} \gamma_{1},|\delta \alpha|_{2}<\frac{1}{2} \gamma_{1}$, we obtain that the first equation in (3.20) has a unique solution in $\delta \phi$, and this solution can be written in the form

$$
\delta \phi=G\left(\alpha_{0}+\delta \alpha\right) Q^{*} a \delta \psi-G\left(\alpha_{0}+\delta \alpha\right) \phi_{0} \delta \alpha+G\left(\alpha_{0}+\delta \alpha\right) f_{1} .
$$

We substitute this solution into the second equation in (3.20), and we get

$$
\begin{aligned}
\phi_{0} & \cdot G\left(\alpha_{0}+\delta \alpha\right) \phi_{0} \delta \alpha-\frac{1}{\lambda} \delta \alpha+\phi_{0} \cdot G\left(\alpha_{0}+\delta \alpha\right) Q^{*} a \delta \psi+\phi_{0} \cdot G\left(\alpha_{0}+\delta \alpha\right) f_{1} \\
& +\frac{1}{2}\left(G\left(\alpha_{0}+\delta \alpha\right) Q^{*} a \delta \psi+G\left(\alpha_{0}+\delta \alpha\right) f_{1}\right)^{2}-\left(G\left(\alpha_{0}+\delta \alpha\right) Q^{*} a \delta \psi\right. \\
& \left.+G\left(\alpha_{0}+\delta \alpha\right) f_{1}\right) \cdot G\left(\alpha_{0}+\delta \alpha\right) \phi_{0} \delta \alpha+\frac{1}{2}\left(G\left(\alpha_{0}+\delta \alpha\right) \phi_{0} \delta \alpha\right)^{2}=\frac{1}{\lambda} \alpha_{1}
\end{aligned}
$$

It is a non-linear equation for $\delta \alpha$, with $\delta \psi, \phi_{0}, \alpha_{0}, f_{1}, \alpha_{1}$ in the space (3.21), where $\delta_{0}, \delta_{1}$ are sufficiently small. To understand a structure of this equation we expand the operator $G\left(\alpha_{0}+\delta_{\alpha}\right)$ up to the second order in $\delta \alpha$ using the identity (3.22). We have

$$
G\left(\alpha_{0}+\delta \alpha\right)=G\left(\alpha_{0}\right)-G\left(\alpha_{0}\right) \delta \alpha G\left(\alpha_{0}\right)+G\left(\alpha_{0}\right) \delta \alpha G\left(\alpha_{0}\right) \delta \alpha G\left(\alpha_{0}+\delta \alpha\right),
$$


and we substitute this expansion into Eq. (3.24). We write the obtained equation in the following form:

$$
G_{0} \delta \alpha+F_{2}(\delta \alpha)=F_{0} .
$$

The first term on the left-hand side is the linear part of Eq. (3.24), and it is given by

$$
\begin{aligned}
G_{0} \delta \alpha= & \phi_{0} \cdot G\left(\alpha_{0}\right) \phi_{0} \delta \alpha+\frac{1}{\lambda} \delta \alpha+\left(\phi_{0}+G\left(\alpha_{0}\right) Q^{*} a \delta \psi+G\left(\alpha_{0}\right) f_{1}\right) \\
& \cdot\left(G\left(\alpha_{0}\right) \delta \alpha G\left(\alpha_{0}\right) Q^{*} a \delta \psi+G\left(\alpha_{0}\right) \delta \alpha G\left(\alpha_{0}\right) f_{1}\right) \\
& +\left(G\left(\alpha_{0}\right) Q^{*} a \delta \psi+G\left(\alpha_{0}\right) f_{1}\right) \cdot G\left(\alpha_{0}\right) \phi_{0} \delta \alpha
\end{aligned}
$$

The second term $F_{2}(\delta \alpha)$ is a sum of terms which are at least of second order in $\delta \alpha$. We can write it explicitly, but a resulting expression is too long, we need only its general properties. It is a sum of terms which have one of the following two forms:

$$
\phi_{0} \cdot G\left(\alpha_{0}\right) f, \quad G\left(\alpha_{0}\right) f \cdot G\left(\alpha_{0}\right) g
$$

multiplied by \pm 1 , or $\pm \frac{1}{2}$, where $f, g$ are equal to one of the functions obtained by applying the operators $I, \delta_{\alpha} G\left(\alpha_{0}\right), \delta \alpha G\left(\alpha_{0}\right) \delta \alpha G\left(\alpha_{0}+\delta \alpha\right)$ to the functions $Q^{*} a \delta \psi, f_{1}, \phi_{0} \delta \alpha$. The terms must satisfy the additional restriction that there are at least two factors $\delta \alpha$. Let us assume that $\delta_{1}, \gamma_{1}$ are sufficiently small, so that $3 B_{0} \delta_{1} \leqq 1, B_{0} \gamma_{1} \leqq 1$. Inspecting the function $F_{2}(\delta \alpha)$ and using the bounds (2.57) in $[2(\mathrm{~b})]$ we get easily the following bound:

$$
\left|F_{2}(\delta \alpha)\right| \leqq 20 B_{0}^{2}|\delta \alpha|_{2}^{2}
$$

The function $F_{2}(\delta \alpha)$ is an analytic function of $\alpha_{0}, \delta \alpha$, and also a second order polynomial in $\phi_{0}, \delta \psi, f_{1}$. Finally, the right-hand side $F_{0}$ in (3.26) is obtained by taking the terms with $\delta \alpha=0$ in (3.24), and it is given by

$$
F_{0}=\phi_{0} \cdot\left(G\left(\alpha_{0}\right) Q^{*} a \delta \psi+G\left(\alpha_{0}\right) f_{1}\right)+\frac{1}{2}\left(G\left(\alpha_{0}\right) Q^{*} a \delta \psi+G\left(\alpha_{0}\right) f_{1}\right)^{2}-\frac{1}{\lambda} \alpha_{1} .
$$

We obtain easily the bound $\left|F_{0}\right|<\frac{3}{2} B_{0} \frac{5}{2} \delta_{1}+\frac{1}{2}\left(B_{0} \frac{5}{2} \delta_{1}\right)^{2}+\delta_{1} \leqq 2 B_{0} \cdot \frac{5}{2} \delta_{1}+\delta_{1}<$ $6 B_{0} \delta_{1}$. The function $F_{0}$ is an analytic function of $\alpha_{0}$, and a second order polynomial in $\phi_{0}, \delta \psi, f_{1}, \alpha_{1}$.

Consider Eq. (3.26). It follows from the properties of $F_{2}, F_{0}$ that its solvability depends entirely on properties of the operator $G_{0}$. We write this operator as

$$
G_{0} \delta \alpha=\phi_{0} \cdot G\left(\alpha_{0}\right) \phi_{0} \delta \alpha+\frac{1}{\lambda} \delta \alpha+R_{1} \delta \alpha
$$

and the operator $R_{1}$ is small. Indeed, from (3.27) we have

$$
\left|R_{1} \delta \alpha\right| \leqq\left(\frac{3}{2}+B_{0} \frac{5}{2} \delta_{1}\right) B_{0}^{2} \frac{5}{2} \delta_{1}|\delta \alpha|_{2}+B_{0} \frac{5}{2} \delta_{1} B_{0} \frac{3}{2}|\delta \alpha|_{2} \leqq 10 B_{0}^{2} \delta_{1}|\delta \alpha|_{2}
$$

The operator $\phi_{0} \cdot G\left(\alpha_{0}\right) \phi_{0}+\frac{1}{\lambda}$ is not small, but $\frac{1}{\lambda}$ may be arbitrarily small, or even 0 in the limiting case $\lambda=+\infty$, and $\phi_{0} \cdot G\left(\alpha_{0}\right) \phi_{0}$ is not well separated from 0 . In fact this operator is close to $G\left(\alpha_{0}\right)$, the difference being small for $\delta_{0}$ small, and the inverse to the operator $G\left(\alpha_{0}\right)$ contains the Laplace operator on the $\eta$-lattice, which is large for $k$ large. Thus we expect that the inverse $G_{0}^{-1}$ is not bounded uniformly 
in $k$. This is a problem, but a closer inspection of terms in Eq. (3.26) shows that they are quite regular, as is clear from (3.28). In particular we may apply the Laplace operator to them, and small terms remain still small. The Laplace operator is a leading term in $G_{0}^{-1}$, so applying $G_{0}^{-1}$ to Eq. (3.26), we should obtain an equation to which a simple form of an inverse mapping theorem could be applied. We shall now explain in detail the above statements, and discuss corresponding bounds. We study first the application of the operator $G^{-1}\left(\alpha_{0}\right)$ to expressions of the form (3.28). Let us write a general formula for the Laplace operator of a product of two functions. Dropping the superscript $\eta$ we have

$$
(\Delta f g)(x)=(\Delta f)(x) g(x)+\sum_{b \in s t(x)} \partial f(b) \partial g(b)+f(x)(\Delta g)(x),
$$

where $\operatorname{st}(x)=\left\{b=\left\langle b_{-}, b_{+}\right\rangle \subset T: b_{-}=x\right\}$. The same formula holds for the operator with Dirichlet boundary conditions on a set $\Omega$; we have to put at least one of the functions $f, g$ equal to 0 on $\Omega^{c}$, and take $x \in \Omega$. It is convenient to write the above formula in an operator form. Introduce a symmetrized product of gradients of two functions as

$$
(\nabla f \cdot \nabla g)(x)=\frac{1}{2} \sum_{b \in s t(x)} \partial f(b) \partial g(b)
$$

then we have

$$
\Delta f g=(\Delta f) g+2 \nabla f \cdot \nabla g+f(\Delta g) \text {. }
$$

Let us apply the operator $G^{-1}\left(\alpha_{0}\right)$ to the expression $\phi_{0} \cdot G\left(\alpha_{0}\right) f$. The function $G\left(\alpha_{0}\right) f$ is extended naturally by 0 on $\Omega_{1}^{c}$, so we have

$$
\begin{gathered}
G^{-1}\left(\alpha_{0}\right)\left(\phi_{0} \cdot G\left(\alpha_{0}\right) f\right)=\left(-\Delta \phi_{0}\right) \cdot G\left(\alpha_{0}\right) f-2 \nabla \phi_{0} \cdot \nabla G\left(\alpha_{0}\right) f \\
+Q^{*} a Q\left(\phi_{0} \cdot G\left(\alpha_{0}\right) f\right)-\phi_{0} \cdot Q^{*} a Q G\left(\alpha_{0}\right) f+\phi_{0} \cdot f .
\end{gathered}
$$

Using the assumptions (3.21) and the bounds for $G\left(\alpha_{0}\right)$ we have

$$
\begin{aligned}
\mid G^{-1}\left(\alpha_{0}\right)\left(\left.\phi_{0} \cdot G\left(\alpha_{0}\right) f\right|_{2}\right. & \leqq \delta_{0} B_{0}|f|_{2}+2 d \delta_{0} B_{0}|f|_{2}+\frac{3}{2} d \delta_{0} B_{0}|f|_{2}+\frac{3}{2}|f|_{2} \\
& \leqq 2|f|_{2}, \quad \text { for } \delta_{0} \text { sufficiently small }
\end{aligned}
$$

Similarly for the expression $G\left(\alpha_{0}\right) f \cdot G\left(\alpha_{0}\right) g$ we have

$$
\begin{gathered}
G^{-1}\left(\alpha_{0}\right)\left(G\left(\alpha_{0}\right) f \cdot G\left(\alpha_{0}\right) g\right)=\left(-\Delta G\left(\alpha_{0}\right) f\right) \cdot G\left(\alpha_{0}\right) g-2 \nabla G\left(\alpha_{0}\right) f \cdot \nabla G\left(\alpha_{0}\right) g \\
+Q^{*} a Q\left(G\left(\alpha_{0}\right) f \cdot G\left(\alpha_{0}\right) g\right)-G\left(\alpha_{0}\right) f \cdot Q^{*} a Q G\left(\alpha_{0}\right) g+G\left(\alpha_{0}\right) f \cdot g, \\
\text { hence } \mid G^{-1}\left(\alpha_{0}\right)\left(\left.G\left(\alpha_{0}\right) f \cdot G\left(\alpha_{0}\right) g\right|_{2} \leqq\left(B_{0}^{2}+2 d B_{0}^{2}+\frac{3}{2} d B_{0}^{2}+B_{0}\right)|f|_{2}|g|_{2}\right. \\
\leqq 5 d B_{0}^{2}|f|_{2}|g|_{2} .
\end{gathered}
$$

Now let us recall the structure of the function $F_{2}(\delta \alpha)$ described after (3.28). From the description and the above bounds it follows easily that there exists an absolute constant $O(1)$ such that

$$
\left|G^{-1}\left(\alpha_{0}\right) F_{2}\left(\alpha_{0}\right)\right|_{2} \leqq O(1) d B_{0}^{2}|\delta \alpha|_{2}^{2}
$$


if $\delta_{0}, \delta_{1}, \gamma_{1}$ are sufficiently small. For the function $F_{0}$ given by (3.29) we obtain

$$
\begin{aligned}
\left|G^{-1}\left(\alpha_{0}\right) F_{0}\right|_{2} & <5 \delta_{1}+\frac{1}{2} 5 d B_{0}^{2}\left(\frac{5}{2} \delta_{1}\right)^{2}+\left(4 d L+\frac{5}{2}+\delta_{0}\right) \delta_{1}<5 d L \delta_{1}+16 d B_{0}^{2} \delta_{1}^{2} \\
& \leqq 6 d L \delta_{1} \text {, if } \delta_{1} \text { is sufficiently small, e.g. } 16 d B_{0}^{2} \delta_{1} \leqq 1
\end{aligned}
$$

For the operator $R_{1}$ we obtain

$$
\begin{aligned}
\left|G^{-1}\left(\alpha_{0}\right) R_{1} \delta \alpha\right|_{2} \leqq & 2|\delta \alpha|_{2} B_{0} \frac{5}{2} \delta_{1}+5 d B_{0}^{2}\left(\frac{5}{2} \delta_{1}|\delta \alpha|_{2} B_{0} \frac{5}{2} \delta_{1}\right) \\
& +\frac{5}{2} \delta_{1} \frac{3}{2}|\delta \alpha|_{2} \leqq 20 d B_{0}^{2} \delta_{1}|\delta \alpha|_{2} .
\end{aligned}
$$

Finally, for the most important first two terms on the right-hand side of (3.30) we have

$$
\begin{aligned}
G^{-1} & \left(\alpha_{0}\right)\left(\phi_{0} \cdot G\left(\alpha_{0}\right) \phi_{0} \delta \alpha+\frac{1}{\lambda} \delta \alpha\right) \\
= & \left(-\Delta \phi_{0}\right) \cdot G\left(\alpha_{0}\right) \phi_{0} \delta \alpha-2 \nabla \phi_{0} \cdot \nabla G\left(\alpha_{0}\right) \phi_{0} \delta \alpha+\left[Q^{*} a Q, \phi_{0}\right] \cdot G\left(\alpha_{0}\right) \phi_{0} \delta \alpha \\
& +\left(\phi_{0}^{2}-1\right) \delta \alpha+\frac{\alpha_{0}}{\lambda} \delta \alpha+\delta \alpha+\frac{1}{\lambda}\left(-\Delta_{\Omega_{1}}^{D}+Q^{*} a Q+v\right) \delta \alpha \\
= & R_{2} \delta \alpha+\delta \alpha+\frac{1}{\lambda}\left(-\Delta_{\Omega_{1}}^{D}+Q^{*} a Q+v\right) \delta \alpha
\end{aligned}
$$

where the last equality defines the operator $R_{2}$, and the symbol [A,B] denotes the commutator of two operators, i.e. $[A, B]=A B-B A$. The operator $R_{2}$ is small, it satisfies the bound

$$
\begin{aligned}
\left|R_{2} \delta \alpha\right|_{2} & \leqq \delta_{0} B_{0} \frac{3}{2}|\delta \alpha|_{2}+2 d \delta_{0} B_{0} \frac{3}{2}|\delta \alpha|_{2}+\frac{3}{2} d \delta_{0} B_{0} \frac{3}{2}|\delta \alpha|_{2}+2 \delta_{0}|\delta \alpha|_{2} \\
& \leqq 6 d B_{0} \delta_{0}|\delta \alpha|_{2}
\end{aligned}
$$

We have used here, as in (3.35), the assumption $\lambda \eta^{2} \geqq 1$. We have also assumed that $B_{0}$ is not small, e.g. $B_{0} \geqq 4$.

The above analysis shows that applying the operator $G^{-1}\left(\alpha_{0}\right)$ to Eq. (3.26), we obtain the equivalent equation

$$
\begin{gathered}
\delta \alpha+\frac{1}{\lambda}\left(-\Delta_{\Omega_{1}}^{D}+Q^{*} a Q+v\right) \delta \alpha+R_{2} \delta \alpha+G^{-1}\left(\alpha_{0}\right) R_{1} \delta \alpha+G^{-1}\left(\alpha_{0}\right) F_{2}(\delta \alpha) \\
=G^{-1}\left(\alpha_{0}\right) F_{0}
\end{gathered}
$$

with terms satisfying the bounds (3.38), (3.36), (3.34), (3.35) correspondingly. We have yet to study the first two linear operators above, i.e. the operator

$$
1+\frac{1}{\lambda}\left(-\Delta_{\Omega_{1}}^{D}+Q^{*} a Q+v\right)=\frac{1}{\lambda}\left(-\Delta_{\Omega_{1}}^{D}+Q^{*} a Q+v+\lambda\right)=\frac{1}{\lambda} G^{-1}(\lambda) .
$$

It is obviously an invertible operator and its inverse is equal to $\lambda G(\lambda)$. The inverse has the same properties as the operators $G(\alpha)$, in particular it is bounded in the same scaled supremum norms (3.17), the only problem is uniform boundedness in 
$\lambda$. This is actually very simple if $\lambda \eta^{2}$ is large enough, e.g. iff $\lambda \eta^{2} \geqq 8 d L^{2}+2$. Then we can write the operator (3.40) in the rescaled form

$$
1+\frac{1}{\lambda \eta^{2}}\left(-\Delta_{\Omega_{1}}^{D, 1}+Q^{*} a \eta^{2} Q+v \eta^{2}\right)
$$

and we can consider it as a small perturbation of the identity. Indeed, the norm of the second operator above in the space with the norm $|\cdot|_{2}$ can be bounded by $\frac{1}{\lambda \eta^{2}}\left(4 d L^{2}+\frac{3}{2} L^{-2}+L^{-2}\right)<\frac{1}{\lambda \eta^{2}}\left(4 d L^{2}+1\right) \leqq \frac{1}{2}$, hence the inverse operator is given by the convergent Neumann series, and its norm in the same space can be bounded by 2 . The norm of the operator $\lambda G(\lambda)$ is bounded uniformly for all positive $\lambda$, i.e. we have

$$
|\lambda G(\lambda) f|_{2} \leqq O(1)|f|_{2}
$$

with a constant $O(1)$ depending on $d$ only, and independent of $\lambda>0$. We need this bound for $\lambda \geqq \eta^{-2}$, and we will prove it later in the next paper, together with its localization expansion. A simple proof can be given using the localization. Now we apply the inverse operator to Eq. (3.39) and we obtain the following equation:

$$
\begin{aligned}
\delta \alpha+\lambda G(\lambda) R_{2} \delta \alpha+ & \lambda G(\lambda) G^{-1}\left(\alpha_{0}\right) R_{1} \delta \alpha+\lambda G(\lambda) G^{-1}\left(\alpha_{0}\right) F_{2}(\delta \alpha) \\
& =\lambda G(\lambda) G^{-1}\left(\alpha_{0}\right) F_{0} .
\end{aligned}
$$

It can be written in the simpler form,

$$
\delta \alpha+H_{1} \delta \alpha+H_{2}(\delta \alpha)=H_{0},
$$

with an obvious identification of the terms. Let us summarize basic properties of these terms. We have shown that $H_{1} \delta \alpha, H_{2}(\delta \alpha), H_{0}$ are analytic functions of $\delta \psi, \phi_{0}, \alpha_{0}, f_{1}, \alpha_{1}$ on domains (3.21) with $\delta_{0}, \delta_{1}$ sufficiently small, and of $\delta \alpha$ on the domain $\left\{|\delta \alpha|_{2}<\frac{1}{2} \gamma_{1}\right\}$. There exists a positive constant $B_{1}$, depending on $B_{0}, d, L$ only (hence on $d, L$ only), such that these functions satisfy the bounds

$$
\left|H_{1} \delta \alpha\right|_{2} \leqq B_{1}\left(\delta_{0}+\delta_{1}\right)|\delta \alpha|_{2},\left|H_{2}(\delta \alpha)\right|_{2} \leqq B_{1}|\delta \alpha|_{2}^{2},\left|H_{0}\right|_{2}<B_{1} \delta_{1}
$$

It is easy now to analyze Eq. (3.43) for small $\delta \alpha$. The function of $\delta \alpha$ on the left-hand side defines an invertible mapping on a neighborhood of 0 , because the sum of the second and third terms defines a contractive mapping on such a neighborhood. More precisely it is a contraction on the domain $\left\{|\delta \alpha|_{2}<c\right\}$ with a contraction factor $\frac{1}{2}$ if $B_{1}\left(\delta_{0}+\delta_{1}+c\right) \leqq \frac{1}{2}$. Then there exists the inverse mapping defined on the domain $\left|H_{0}\right|_{2}<\frac{1}{2} c$, or equivalently Eq. (3.43) has a unique solution $\delta \alpha$ in $\left\{|\delta \alpha|_{2}<c\right\}$ if $\left|H_{0}\right|_{2}<\frac{1}{2} c$. This solution can be constructed as a limit of a sequence of successive approximations, so it is an analytic function of $\delta \psi, \phi_{0}, \alpha_{0}, f_{1}, \alpha_{1}$, and it satisfies the bound $|\delta \alpha|_{2} \leqq 2\left|H_{0}\right|_{2}$. Proofs of the above statements are quite simple and standard; they can be found in many places. We formulate the conclusions in the lemma.

Lemma 3.2. Consider Eq. (3.43) and assume that its terms are analytic functions of $\delta \psi, \phi_{0}, \alpha_{0}, f_{1}, \alpha_{1}$ and defined on the domains (3.21) for $\delta_{0}, \delta_{1}$ sufficiently small, and of $\delta \alpha$ defined on the domain $\left\{|\delta \alpha|_{2}<\frac{1}{2} \gamma_{1}\right\}$. Assume further that they satisfy the bounds (3.44). Then there exists a positive small constant $c_{6}$ such, that if $\delta_{0} \leqq c_{6}, 2 B_{1} \delta_{1} \leqq c_{6}$, then Eq. (3.43) has a unique solution $\delta \alpha$ in the domain 
$\left\{|\delta \alpha|_{2}<c_{6}\right\}$, and the solution is an analytic function of $\delta \psi, \phi_{0}, \alpha_{0}, f_{1}, \alpha_{1}$ satisfying the bound

$$
|\delta \alpha|_{2} \leqq 2\left|H_{0}\right|_{2}<2 B_{1} \delta_{1}
$$

The above lemma completes the analysis of Eq. (3.20). Under the assumptions of the lemma on $\delta_{0}, \delta_{1}$ this system of equations has a unique solution $\delta \phi, \delta \alpha$ in the space of all configurations satisfying only the condition $|\delta \alpha|_{2}<c_{6}$. The solution $\delta \alpha$ satisfies (3.45), and the solution $\delta \phi$ is given by the formula (3.23), therefore it has regularity properties determined by the properties of $G(\alpha)$ discussed after (3.22). In particular we have

$$
\begin{gathered}
|\delta \phi| \leqq B_{0} \frac{3}{2}|\delta \psi|+B_{0} \frac{3}{2}|\delta \alpha|_{2}+B_{0}\left|f_{1}\right|_{2}<B_{0} \frac{3}{2} \delta_{1}+3 B_{0} B_{1} \delta_{1}+B_{0} \delta_{1}<4 B_{0} B_{1} \delta_{1} \\
\left|\partial^{\eta} \delta \phi\right|_{1}<4 B_{0} B_{1} \delta_{1},\left|\partial^{\eta} \delta \phi\right|_{(\alpha), 2-\alpha}<C_{\alpha} 4 B_{0} B_{1} \delta_{1} \\
\left|\Delta_{\Omega_{1}}^{D, \eta} \delta \phi\right|_{2}<4 B_{0} B_{1} \delta_{1}
\end{gathered}
$$

where $0<\alpha \leqq 1$, and $C_{\alpha}$ is a universal constant depending on $\alpha$ and $d$ only, for example it may be chosen in the form $O(1) \alpha^{-d-1}$. We formulate the results of this discussion in the following proposition.

Proposition 3.1. There exist positive constants $c_{6}, K_{2}$ depending on $d, L$ only, such that for $\delta \psi, \phi_{0}, \alpha_{0}, f_{1}, \alpha_{1}$ in a domain (3.21) with $\delta_{0} \leqq c_{6}, K_{2} \delta_{1} \leqq c_{6}$ the system of equations (3.20) has a unique solution in the space of all configurations $\delta \phi, \delta \alpha$ satisfying the restriction $|\delta \alpha|_{2}<c_{6}$ only. The solution is an analytic function of $\delta \psi, \phi_{0}, \alpha_{0}, f_{1}, \alpha_{1}$ satisfying the bounds

$$
\begin{gathered}
|\delta \phi|<K_{2} \delta_{1},\left|\partial^{\eta} \delta \phi\right|_{1}<K_{2} \delta_{1},\left|\partial^{\eta} \delta \phi\right|_{(\alpha), 2-\alpha}<C_{\alpha} K_{2} \delta_{1} \text { for } 0<\alpha \leqq 1 \\
\left|\Delta_{\Omega_{1}}^{D, \eta} \delta \phi\right|_{2}<K_{2} \delta_{1},|\delta \alpha|_{2}<K_{2} \delta_{1}
\end{gathered}
$$

This proposition is one of the main technical results of the paper. It will be used several times in the future, now we apply it to conclude the proof of Proposition 1.1. We have constructed the approximate solution $\phi_{0}, \alpha_{0}$ by the formulas (3.2), (3.3), with properties and bounds described in Lemma 3.1. The corrections $\delta \phi, \delta \alpha$ satisfy Eq. (3.19) with $\delta \psi=0$ and $f_{0}$ given by the second formula in (3.3), hence Eq. (3.20) with $\delta \psi=0, f_{1}=-f_{0}$ and $\alpha_{1}=0$. By Lemma 3.1 the configurations $\delta \psi=0, \phi_{0}, \alpha_{0}, f_{1}=-f_{0}, \alpha_{1}=0$ belong to the domain (3.21) with $\delta_{0}=C_{3} \delta$, $\delta_{1}=C_{3} \delta$ if $\delta, \varepsilon$ satisfy the assumptions of Proposition 2.1 , i.e. $0<\delta \leqq \varepsilon, v \varepsilon^{2} \leqq \delta^{2}$, $\delta \leqq c_{4}$, and $\varepsilon$ is sufficiently small. By Proposition 3.1 Eq. (3.20) in this case have a unique solution if $C_{3} \delta \leqq c_{6}, K_{2} C_{3} \delta \leqq c_{6}$. The uniqueness holds on the space of all configurations $\delta \phi, \delta \alpha$ satisfying the only restriction $|\delta \alpha|_{2}<c_{6}$. This solution determines a solution of the basic equations (1.14) by the formulas (3.18). It is easy to see that the constructed solution of Eq. (1.14) is unique in the space of configurations $\phi, \alpha$ satisfying the only restriction $|\alpha|_{2}<\frac{1}{2} c_{6}$, assuming that $C_{3} \delta \leqq \frac{1}{2} c_{6}$. Indeed, if $\phi, \alpha$ satisfies (1.14) and $|\alpha|_{2}<\frac{1}{2} c_{6}$, then $\delta \phi=\phi-\phi_{0}, \delta \alpha=\alpha-\alpha_{0}$ satisfies the system (3.20), and $|\delta \alpha|_{2} \leqq|\alpha|_{2}+\left|\alpha_{0}\right|_{2}<\frac{1}{2} c_{6}+C_{3} \delta \leqq c_{6}$. Such a solution is unique. Let us assume that $\frac{1}{2} c_{6}$ is smaller than the restriction on $\varepsilon$, 
and let us take $c_{0}=\frac{1}{2} c_{6}, c_{1}=\min \left\{c_{4},\left(2 K_{2}+1\right)^{-1} C_{3}^{-1} c_{6}\right\}$. If $\delta, \varepsilon$ satisfy the conditions $0<\delta \leqq \varepsilon \leqq c_{0}, v \varepsilon^{2} \leqq \delta^{2}, \delta \leqq c_{1}$, then the assumptions of both Propositions 2.1 and 3.1 are satisfied, and we have constructed the solution of the system (1.14), which is unique in the space of configurations $\phi, \alpha$ satisfying the restriction $|\alpha|_{2}<c_{0}$. By Lemma 3.1 and (3.47) this solution satisfies the bounds (1.15), (1.16) of Proposition 1.1 with the constant $K_{1}$ replaced by $\left(2 K_{2}+1\right) C_{3}$. In fact it satisfies all of them, except the last one in (1.15), with the constant $\left(K_{2}+1\right) C_{3}$. The solution is also an analytic function of $\left(\psi+\psi^{\prime}, h+h^{\prime}\right)$. We take $K_{1}=\left(2 K_{2}+1\right) C_{3}$. It follows from the above that we have proved all the statements of Proposition 1.1 , except the one on the relation of real solutions to solutions of the variational problem (1.6) or (1.9). A real solution, i.e. the solution corresponding to a real configuration $(\psi, h) \in \tilde{\Psi}(\delta)$, is a critical point of the function (1.4) or (1.7), and the only one in the domain $\left\{(\phi, \alpha):|\alpha|_{2}<c_{0}\right\}$. The solution $\alpha$ is obviously a maximum of the function (1.7) in $\alpha$, so we have to show that $\phi$ is a minimum of (1.4). Let us expand the function (1.4) around the solution $\phi$, i.e. substitute $\phi+\delta \phi$ in the place of $\phi$ in (1.4), taking $\delta \phi=0$ on $\Omega_{1}^{c}$. We get

$$
\begin{aligned}
A(\psi, \phi+\delta \phi)= & A(\psi, \phi)+\left[\frac{1}{2}\left\langle\delta \phi,\left(-\Delta_{\Omega_{1}}^{D, \eta}+Q^{*} a Q+v+\alpha+\lambda \phi \otimes \phi\right) \delta \phi\right\rangle\right. \\
& \left.+\frac{\lambda}{2}\langle\delta \phi,(\phi \cdot \delta \phi) \delta \phi\rangle+\frac{\lambda}{8}\left\||\delta \phi|^{2}\right\|^{2}\right]
\end{aligned}
$$

where we have used the variational equations (1.10). The quadratic form in $\delta \phi$ above is positive definite, so $\phi$ is a minimum of the function (1.4). Notice that it is the only critical point of (1.4) in the domain $\left\{\phi:\left|\frac{\lambda}{2}\left(|\phi|^{2}-1\right)\right|_{2}<c_{0}\right\}$. Thus we have completed the proof of Proposition 1.1.

Let us denote the solution of Eq. (1.14) by $\phi_{k}, \alpha_{k}$ for simplicity. It is an analytic function of $\left(\psi+\psi^{\prime}, h+h^{\prime}\right)$ on $\tilde{\Psi}^{c}\left(c_{1}, c_{0}\right)$, and in the future we will need its expansions around some fixed configurations. We write

$$
\begin{gathered}
\phi_{k}\left(\psi+\psi^{\prime}+\delta \psi, h+h^{\prime}\right)=\phi_{k}\left(\psi+\psi^{\prime}, h+h^{\prime}\right)+\delta \phi_{k}(\delta \psi), \delta \psi=0 \text { on } \Omega_{1}^{c}, \\
\alpha_{k}\left(\psi+\psi^{\prime}+\delta \psi, h+h^{\prime}\right)=\alpha_{k}\left(\psi+\psi^{\prime}, h+h^{\prime}\right)+\delta \alpha_{k}(\delta \psi),
\end{gathered}
$$

and we obtain that $\delta \phi_{k}, \delta \alpha_{k}$ satisfy Eq. (3.20) with $\phi_{0}=\phi_{k}\left(\psi+\psi^{\prime}, h+h^{\prime}\right), \alpha_{0}=$ $\alpha_{k}\left(\psi+\psi^{\prime}, h+h^{\prime}\right), f_{1}=0, \alpha_{1}=0$. The configurations $\delta \psi, \phi_{k}, \alpha_{k}, 0,0$ belong to the domain (3.21) if $|\delta \psi|<\delta_{1}, K_{1} c_{1} \leqq \delta_{0}$, in particular if $\delta_{0}=c_{6}$, because $K_{1} c_{1} \leqq c_{6}$. If $K_{2} \delta_{1} \leqq c_{6}$, then the assumptions of Proposition 3.1 are satisfied and we obtain all the conclusions of this theorem. For future reference we formulate the above in the following corollary.

Corollary 3.1. Under the assumptions of Proposition 1.1 the functions $\phi_{k}(\psi+$ $\left.\psi^{\prime}+\delta \psi, h+h^{\prime}\right), \alpha_{k}\left(\psi+\psi^{\prime}+\delta \psi, h+h^{\prime}\right)$ are analytic functions of $\left(\psi+\psi^{\prime}, h+h^{\prime}\right)$ on the domains $\tilde{\Psi}^{c}(\delta, \varepsilon)$, and of $\delta \psi$ on neighborhoods of 0 . They have analytic extensions in $\delta \psi$ onto the domains $\left\{|\delta \psi|<\delta_{1}\right\}$ with $K_{2} \delta_{1} \leqq c_{6}$. The functions $\delta \phi_{k}, \delta \alpha_{k}$ defined by (3.49) satisfy Eq. (3.20) with $\phi_{0}=\phi_{k}\left(\psi+\psi^{\prime}, h+h^{\prime}\right)$, $\alpha_{0}=\alpha_{k}\left(\psi+\psi^{\prime}, h+h^{\prime}\right), f_{1}=0, \alpha_{1}=0$, they are analytic functions of $\delta \psi, \phi_{k}, \alpha_{k}$, and they satisfy the bounds (3.47).

In the above constructions and proofs we have used extensively the special form of the action (1.4), most importantly the fact that the third term is the $L^{2}$-norm 
of $|\phi|^{2}-1$. This leads to the function (1.7) and to especially simple variational equations (1.10). This is not really important and we can develop the whole theory and obtain the same results for more general actions. We can also apply other methods of analyzing the problem. Now we would like to sketch very briefly an idea of another method applied to some class of actions generalizing (1.4). We consider a simple generalization obtained by replacing the third term on the righthand side of (1.4) by a term with a more general potential function. We want to preserve the invariance with respect to the orthogonal group, and to have the set of minima equal to the unit sphere, hence we assume that the potential has the form $V\left(|\phi|^{2}-1\right)$, where $V(z)$ is an analytic function on a neighborhood of the real axis, whose expansion at $z=0$ starts with a second order term. We have to assume also that this term dominates the rest of the expansion on some bounded set. We consider the following action:

$$
\begin{aligned}
A_{k}(\psi, \phi)= & \frac{1}{2}\langle\psi-Q \phi, a(\psi-Q \phi)\rangle+\frac{1}{2}\left\|\partial^{\eta} \phi\right\|^{2} \\
& +\frac{1}{2}\left\langle V\left(|\phi|^{2}-1\right), 1\right\rangle+\frac{1}{2} v\|\phi-h\|^{2} .
\end{aligned}
$$

We would like to cover again the case when the derivative $V^{\prime \prime}(0)$ may be large. We follow at first the steps of the previous proof: we consider the constant configurations case, and then we construct the approximate solution $\phi_{0}$. The variational equations in this case have again the form (1.10), but the second equation is replaced by the equation $\alpha=V^{\prime}\left(|\phi|^{2}-1\right)$, and we define correspondingly the configurations $f_{0}, \alpha_{0}$, i.e. $\alpha_{0}=V^{\prime}\left(\left|\phi_{0}\right|^{2}-1\right)$ and $f_{0}$ by the second equation in (3.3). We expand $A_{k}(\psi, \phi)$ around $\phi_{0}$, and we get

$$
\begin{aligned}
A_{k}(\psi, \phi)= & A_{k}\left(\psi, \phi_{0}\right)+\left\langle\delta \phi, f_{0}\right\rangle+\frac{1}{2}\left\langle\delta \phi,\left(-\Delta_{\Omega_{1}}^{D, \eta}+Q^{*} a Q+v+\alpha_{0}\right) \delta \phi\right\rangle \\
& +\frac{1}{2}\left\langle V_{2}\left(\left|\phi_{0}\right|^{2}-1,2 \phi_{0} \cdot \delta \phi+(\delta \phi)^{2}\right),\left(2 \phi_{0} \cdot \delta \phi+|\delta \phi|^{2}\right)^{2}\right\rangle
\end{aligned}
$$

where

$$
V_{2}(u, v)=\int_{0}^{1} d t(1-t) V^{\prime \prime}(u+t v) .
$$

We introduce variables $\chi, \phi^{\prime}$ such that $\delta \phi=\phi_{0} \chi+\phi^{\prime}, \chi$ is real valued, $\phi^{\prime}$ has values in $\mathbb{R}^{N}$ and $\phi_{0} \cdot \phi^{\prime}=0$. Then $2 \phi_{0} \cdot \delta \phi+|\delta \phi|^{2}=2\left|\phi_{0}\right|^{2}\left(\chi+\frac{1}{2} \chi^{2}\right)+\left|\phi^{\prime}\right|^{2}$, and we make a non-linear change of variables $\chi=\chi\left(\chi^{\prime}, \phi^{\prime}\right)$ such that $2\left|\phi_{0}\right|^{2}\left(\chi+\frac{1}{2} \chi^{2}\right)+$ $\left|\phi^{\prime}\right|^{2}=2\left|\phi_{0}\right|^{2} \chi^{\prime}$. From this we get

$$
\chi=\left(1+2 \chi^{\prime}-\frac{\left|\phi^{\prime}\right|^{2}}{\left|\phi_{0}\right|^{2}}\right)^{\frac{1}{2}}-1=\chi^{\prime}+h_{2}\left(\chi^{\prime},\left|\phi^{\prime}\right|^{2}\right),
$$

where $h_{2}\left(\chi^{\prime},\left|\phi^{\prime}\right|^{2}\right)$ is an analytic function of at least second order on a neighborhood of $\chi^{\prime}=0, \phi^{\prime}=0$. This change of variables yields the following form of the expansion:

$$
\begin{gathered}
A_{k}\left(\psi, \phi_{0}\right)+\left\langle\phi_{0} \chi^{\prime}+\phi^{\prime \prime}+\phi_{0} h_{2}\left(\chi^{\prime},\left|\phi^{\prime}\right|^{2}\right), f_{0}\right\rangle+\frac{1}{2}\left\langle\phi_{0} \chi^{\prime}+\phi^{\prime}+\phi_{0} h_{2}\left(\chi^{\prime},\left|\phi^{\prime}\right|^{2}\right),\right. \\
\left.G^{-1}\left(\alpha_{0}\right)\left(\phi_{0} \chi^{\prime}+\phi^{\prime}+\phi_{0} h_{2}\left(\chi^{\prime},\left|\phi^{\prime}\right|^{2}\right)\right)\right\rangle+2\left\langle V_{2}\left(\left|\phi_{0}\right|^{2}-1,2\left|\phi_{0}\right| \chi^{\prime}\right),\left|\phi_{0}\right|^{4} \chi^{\prime 2}\right\rangle .
\end{gathered}
$$


The function $V_{2}\left(\left|\phi_{0}\right|^{2}-1,2\left|\phi_{0}\right| \chi^{\prime}\right)$ has values close to $\frac{1}{2} V^{\prime \prime}(0)$ for $\left|\phi_{0}\right|^{2}-1$ and $2\left|\phi_{0}\right| \chi^{\prime}$ small enough, a bit more precisely we assume

$$
\begin{aligned}
\left|V_{2}\left(\left|\phi_{0}\right|^{2}-1,2\left|\phi_{0}\right| \chi^{\prime}\right)-\frac{1}{2} V^{\prime \prime}(0)\right| & \leqq O\left(V^{\prime \prime}(0)\right)\left(\left.|| \phi_{0}\right|^{2}-1|+| \chi^{\prime} \mid\right) \\
& \leqq O(1)\left(\left|\alpha_{0}\right|+V^{\prime \prime}(0)\left|\chi^{\prime}\right|\right), V^{\prime \prime}(0)>0 .
\end{aligned}
$$

We make a last change of variables, the simple rescaling $\chi^{\prime}=\lambda^{-\frac{1}{2}} \chi$, where $\lambda=2 V^{\prime \prime}(0)$. It is easy to see that we obtain an action in $\chi, \phi^{\prime}$, whose second order terms determine a quadratic form with a positive operator. An inverse to this operator has properties similar to properties of $G\left(\alpha_{0}\right)$. The remaining terms are small in comparison with the quadratic form, so that their contributions to variational equations can be treated as small perturbations. These equations can be solved then again by an application of the inverse mapping theorem. A proof based on the above ideas is as straightforward as the proof given before, but there are more technical details to work out. Nevertheless it was important to present these ideas to give a right perspective on our assumptions on the class of models considered here. We will also use them in the future discussing the first renormalization transformation.

\section{Functions Connecting Minimal Solutions for Two Generating Sets}

In this section we study generalizations of the second variational problem (2.6) in [1]. We consider now two generating sets, one after $j$ steps denoted by $\mathbb{B}_{j}$, and one after $k$ steps denoted by $\mathbb{B}_{k}^{\prime}$. We may identify them with the corresponding sequences of domains $\left\{\Omega_{1}, \ldots, \Omega_{j}\right\}$ and $\left\{\Omega_{1}^{\prime}, \ldots, \Omega_{k}^{\prime}\right\}, j \leqq k$, considered in $\eta$-scale. We assume that

$$
\Omega_{1} \subset \Omega_{1}^{\prime}, \ldots, \Omega_{j} \subset \Omega_{j}^{\prime} \text { and we write } \mathbb{B}_{j} \prec \mathbb{B}_{k}^{\prime}
$$

Let us stress that in the future we will need this geometric setting in full generality described above. We will comment on some applications later, after formulating Proposition 4.1 on the variational problems. To each generating set there corresponds a renormalization transformation which is a composition of the one-step renormalization transformations (1.11) in [1] such that blocks of the transformations of the $n^{\text {th }}$ step are contained in the corresponding $n^{\text {th }}$ domain. We assume here, as we have stated in Sect. 1 that the only changes of constants determining the transformations are given by scaling transformations, more precisely in the $n^{\text {th }}$ step transformations we put the constant $\beta L^{n(d-2)}$ instead of $\beta L^{d-2}$ in (1.11) [1]. This composition is an integral transformation with a Gaussian kernel, and the quadratic form defining this kernel is equal to (1.5) multiplied by $\beta L^{k(d-2)}$, if $k$ is a number of steps. Consider the transformation determined by $\mathbb{B}_{j}$. It is clear by (4.1) that we have to perform some additional transformations to get the one determined by $\mathbb{B}_{k}^{\prime}$. We take the composition of those additional transformations, and we would like to understand its structure and properties. We need them to formulate and investigate the generalization of the problem (2.6) in [1]. 
To study the compositions we start with the following basic formula:

$$
\begin{aligned}
& \int d \psi\left\lceil B(y) \exp \left[-\frac{1}{2} A|\theta-(Q \psi)(y)|^{2}\right] \exp \left[-\frac{1}{2} \sum_{x \in B(y)} B(x)|\psi(x)-f(x)|^{2}\right]\right. \\
& =\text { const. } \exp \left[-\inf _{\psi}\left\{\frac{1}{2} A|\theta-(Q \psi)(y)|^{2}+\frac{1}{2} \sum_{x \in B(y)} B(x)|\psi(x)-f(x)|^{2}\right\}\right] \\
& =\text { const. } \exp \left[-\frac{1}{2} C|\theta-(Q f)(y)|^{2}\right], C=\frac{A}{L^{-d}\left(Q_{\left.\frac{A}{B(\cdot)}\right)}\right)(y)+1},
\end{aligned}
$$

where the constant is equal to the integral above calculated for $\theta=0, f=0$. It can be calculated explicitly in terms of the constants $A, B(x)$, but it is not important; it will be a part of an overall normalization constant for the composition we want to study. The first equality above is quite general, it holds for any Gaussian integral with a linear form added to the quadratic form. The second equality is obtained by calculating and substituting the minimum of the expression in the curly brackets. Let us now discuss applications of this formula. A composition of renormalization transformations from the $n+1^{\text {st }}$ and $n+2^{\text {nd }}$ steps is obtained by taking $A=\beta L^{(n+2)(d-2)} a, B(x)=\beta L^{(n+1)(d-2)} a, \theta=\psi_{n+2}(y), \psi=\psi_{n+1}, f=Q \psi_{n}$, and a result is a transformation given by the kernel (4.2) with $C=\beta L^{(n+2)(d-2)} a_{2}$, $a_{2}=\frac{a}{L^{2}+1}$. It is clear that composing transformations from $r$ steps we obtain a kernel

$$
\text { const. } \exp \left[-\frac{1}{2} \beta L^{(n+r)(d-2)} a_{r}\left|\psi_{n+r}(y)-\left(Q_{r} \psi_{n}\right)(y)\right|^{2}\right] .
$$

Applying (4.2) with $A=\beta L^{(n+r+1)(d-2)} a, B(x)=\beta L^{(n+r)(d-2)} a_{r}, \theta=\psi_{n+r+1}(y)$, $\psi=\psi_{n+r}, f=Q_{r} \psi_{n}$, we obtain the above kernel with $r$ replaced by $r+1$, and $a_{r+1}$ given by the equation

$$
a_{r+1}=\frac{a}{L^{-2} \frac{a}{a_{r}}+1}=\frac{a a_{r}}{L^{-2} a+a_{r}}, \text { hence } a_{r}=a \frac{1-L^{-2}}{1-L^{-2 r}} .
$$

The sequence $a_{r}$ is decreasing and $a_{r} \searrow a_{\infty}=a\left(1-L^{-2}\right)$. The formula (4.3) has been used before to formulate the variational problem (2.6) in [1], and also the definitions (1.4), (1.5) if $r=j, n=0$. It applies also to the problem we consider here if the cube $\Delta_{n+r}(y)$ intersects the set $\Lambda_{n}$ only. Let us recall that if a point $y \in T_{\varepsilon}^{(j)}$, then $\Delta_{j}(y)$ denotes a continuous space cube with a center at $y$ and sides of the length $\varepsilon$. Consider now the case when $\Delta_{n+r+1}(y)$ intersects two sets $\Lambda_{n}, \Lambda_{n+1}$, but each $\Delta_{n+r}(x)$ for $x \in B(y)$ intersects only one of those domains. For $x \in B(y) \cap \Lambda_{n}$ the composition of transformations we study, with blocks contained in $A_{n+r}(x)$, is given by (4.3) with $x$ instead of $y$. The corresponding composition for $x \in B(y) \cap \Lambda_{n+1}$ is given by (4.3) with $n, r, y$ replaced by $n+1, r-1, x$. Now we take these transformations and compose them with the next transformation for the block $B(y), y \in T^{(n+r+1)}$. This composition has a kernel which can be calculated applying (4.2) with $A=\beta L^{(n+r+1)(d-2)} a, B(x)=\beta L^{(n+r)(d-2)} a_{r}$ for $x \in B(y) \cap$ $\Lambda_{n}, B(x)=\beta L^{(n+r)(d-2)} a_{r-1}$ for $x \in B(y) \cap \Lambda_{n+1}, \theta=\psi_{n+r+1}(y), \psi=\psi_{n+r}, f(x)=$ $\left(Q_{r} \psi_{n}\right)(x)$ for $x \in B(y) \cap \Lambda_{n}, f(x)=\left(Q_{r-1} \psi_{n+1}\right)(x)$ for $x \in B(y) \cap \Lambda_{n+1}$. The 
kernel is given by the last expression in (4.2), where the constant $C$ is equal to

$$
\begin{aligned}
C & =\beta L^{(n+r+1)(d-2)} \frac{a}{a L^{-2}\left(L^{-d}\left|B(y) \cap \Lambda_{n}\right| \frac{1}{a_{r}}+L^{-d}\left|B(y) \cap \Lambda_{n+1}\right| \frac{1}{a_{r-1}}\right)+1} \\
& =\beta L^{(n+r+1)(d-2)} \frac{1}{L^{-d}\left|B(y) \cap \Lambda_{n}\right| \frac{1}{a_{r+1}}+L^{-d}\left|B(y) \cap \Lambda_{n+1}\right| \frac{1}{a_{r}}} .
\end{aligned}
$$

The quotient above replaces the constant $a_{r+1}$ in the previous case. It is determined by the intersection $B(y) \cap \mathbb{B}_{j}$, or by the intersection $\Delta_{n+r+1}(y) \cap \mathbb{B}_{j}$, and we denote it by $a\left(\Delta_{m+r+1}(y), \mathbb{B}_{j}\right)$. From the monotonicity of the sequence $a_{r}$ we obtain the inequalities

$$
a_{r+1}<a\left(\Delta_{n+r+1}(y), \mathbb{B}_{j}\right)<a_{r} .
$$

The average $(Q f)(y)$ in (4.2) is now equal to the following average of a configuration $\psi$ defined on $\mathbb{B}_{j}$,

$$
Q\left(\Delta_{n+r+1}(y), \mathbb{B}_{j}\right) \psi=\sum_{x \in B(y) \cap \Lambda_{n}} L^{-d}\left(Q_{r} \psi_{n}\right)(x)+\sum_{x \in B(y) \cap \Lambda_{n+1}} L^{-d}\left(Q_{r-1} \psi_{n+1}\right)(x) .
$$

Let us consider a general situation. Take a point $y \in T^{(p)}$ such that $\Delta_{p}(y)$ does not intersect the sets $\Lambda_{n}$ with $n \geqq p$, and take the composition of all the renormalization transformations applied after the transformations determined by $\mathbb{B}_{j}$, and corresponding to blocks contained in the cube $\Delta_{p}(y)$. We assume that the composition is a transformation with a Gaussian kernel of the form

$$
\text { const. } \exp \left[-\frac{1}{2} \beta L^{p(d-2)} a\left(\Delta_{p}(y), \mathbb{B}_{j}\right)\left|\psi_{p}(y)-Q\left(\Delta_{p}(y), \mathbb{B}_{j}\right) \psi\right|^{2}\right],
$$

where the averaging operation applied to $\psi$ is given by the formula

$$
Q\left(\Delta_{p}(y), \mathbb{B}_{j}\right) \psi=\sum_{n=0}^{p-1} \sum_{x \in \Delta_{p}(y) \cap \Lambda_{n}} \frac{\left|\Delta_{n}(x)\right|}{\left|\Delta_{p}(y)\right|} \psi_{n}(x),
$$

and the constant $a\left(\Delta_{p}(y), \mathbb{B}_{J}\right)$ is given by the formula

$$
\frac{1}{a\left(\Delta_{p}(y), \mathbb{B}_{j}\right)}=\sum_{n=0}^{p-1} \sum_{x \in \Delta_{p}(y) \cap \Lambda_{n}} \frac{\left|\Delta_{n}(x)\right|}{\left|\Delta_{p}(y)\right|} \frac{1}{a_{p-n}} .
$$

The constant in front of the exponential in (4.5) is a normalization constant which can be calculated explicitly from the requirement that the integral of (4.5) with respect to the variable $\psi_{p}(y)$ is equal to 1 . The formulas $(4.5)-(4.7)$ coincide with all the formulas obtained in the special cases discussed before, and are easy to prove by induction. Take $y^{\prime} \in T^{(p+1)}$ and such that $\Delta_{p+1}\left(y^{\prime}\right)$ does not intersect the sets $\Lambda_{n}$ with $n \geqq p+1$. If $\Delta_{p+1}\left(y^{\prime}\right)$ intersects $\Lambda_{p}$, then the block $B\left(y^{\prime}\right)$ must be contained in $\Lambda_{p}$, because $\Lambda_{p}$ is a union of large blocks, i.e. blocks of the order $m$, where $L^{m}=M$. In this case the composition determined by $\Delta_{p+1}\left(y^{\prime}\right)$ is just the basic renormalization transformation (4.3) for $n=p, r=1$. Thus we may assume that $\Delta_{p+1}\left(y^{\prime}\right)$, and therefore also $\Delta_{p}(y)$ for $y \in B\left(y^{\prime}\right)$, do not intersect $\Lambda_{n}$ with $n \geqq p$. Then we use the inductive assumptions $(4.5)-(4.7)$ for the cubes $\Delta_{p}(y)$, and we compose the transformations for those cubes with the next renormalization 
transformation for the block $B\left(y^{\prime}\right)$. We calculate the kernal of this composition using the formula (4.2) with $A=\beta L^{(p+1)(d-2)} a, B(y)=\beta L^{p(d-2)} a\left(\Delta_{p}(y), \mathbb{B}_{j}\right), \theta=$ $\psi_{p+1}\left(y^{\prime}\right), \psi=\psi_{p}, f(y)=Q\left(\Delta_{p}(y), \mathbb{B}_{j}\right) \psi, y \in B\left(y^{\prime}\right)$. We obtain the last exponential in (4.2), and the formula for $C$ implies that this constant is equal to $\beta L^{(p+1)(d-2)}$ $a\left(\Delta_{p+1}\left(y^{\prime}\right), \mathbb{B}_{j}\right)$, where $a\left(\Delta_{p+1}\left(y^{\prime}\right), \mathbb{B}_{j}\right)$ is given again by the formula (4.7). In this calculation we have to use also Eq. (4.4). It is also easy to see that the average $(Q f)\left(y^{\prime}\right)$ is equal to $Q\left(\Delta_{p+1}\left(y^{\prime}\right), \mathbb{B}_{J}\right) \psi$ given by (4.6). Thus we have proved the formulas (4.5)-(4.7). From (4.7) we obtain the inequality

$$
a_{p} \leqq a\left(\Delta_{p}(y), \mathbb{B}_{j}\right) \leqq a_{1}=a
$$

It means that all these constants belong to the interval $\left[a\left(1-L^{2}\right), a\right]$, a fact which is important for bounds.

Now we take the generating set $\mathbb{B}_{k}^{\prime}$ and a product of the transformations defined by (4.5) for points of the set. To write this product in a simple and convenient way we introduce some new definitions and notations. We define the averaging operator $Q\left(\mathbb{B}_{k}^{\prime}, \mathbb{B}_{j}\right)$ by

$$
\begin{aligned}
& \left(Q\left(\mathbb{B}_{k}^{\prime}, \mathbb{B}_{j}\right) \psi\right)\left(y^{\prime}\right)=Q\left(\Delta_{p}\left(y^{\prime}\right), \mathbb{B}_{j}\right) \psi \quad \text { if } y^{\prime} \in \Lambda_{p}^{\prime} \cap \Omega_{p}^{(p) c}, \\
& \left(Q\left(\mathbb{B}_{k}^{\prime}, \mathbb{B}_{j}\right) \psi\right)\left(y^{\prime}\right)=\psi_{p}\left(y^{\prime}\right) \text { if } y^{\prime} \in \Lambda_{p}^{\prime} \cap \Omega_{p}^{(p)}=\Lambda_{p}^{\prime} \cap \Lambda_{p},
\end{aligned}
$$

for $p=0,1, \ldots, k$, where we take now $\Lambda_{0}^{\prime}=\Omega_{1}^{\prime c}, \Lambda_{0}=\Omega_{1}^{c}$. This operator transforms functions $\psi$ defined on $\mathbb{B}_{j} \cup \Lambda_{0}$ into functions defined on $\mathbb{B}_{k}^{\prime} \cup \Lambda_{0}^{\prime}$. Next, we define a multiplication operator $a\left(\mathbb{B}_{k}^{\prime}, \mathbb{B}_{j}\right)$ on a space of functions defined on the set $\mathbb{B}_{k}^{\prime} \cup \Lambda_{0}^{\prime}$ by the function

$$
\begin{aligned}
& a\left(y^{\prime} ; \mathbb{B}_{k}^{\prime}, \mathbb{B}_{j}\right)=a\left(\Delta_{p}\left(y^{\prime}\right), \mathbb{B}_{j}\right)\left(L^{p} \eta\right)^{-2} \text { if } y^{\prime} \in \Lambda_{p}^{\prime} \cap \Omega_{p}^{(p) c}, \\
& a\left(y^{\prime} ; \mathbb{B}_{k}^{\prime}, \mathbb{B}_{j}\right)=\text { arbitrary number if } y^{\prime} \in \Lambda_{p}^{\prime} \cap \Lambda_{p} .
\end{aligned}
$$

Notice that the above definition depends on the scale of the original lattice, and is rescaled properly together with the lattice. Further, let us denote by $T\left(\mathbb{B}_{n}\right)$ the composition of the renormalization transformations determined by a generating set $\mathbb{B}_{n}$. It has a Gaussian kernel with a quadratic form equal to a corresponding form (1.5) multiplied by $\beta L^{n(d-2)}$. Finally, we denote by $T\left(\mathbb{B}_{k}^{\prime}, \mathbb{B}_{j}\right)$ the composition of the renormalization transformations applied after $T\left(\mathbb{B}_{j}\right)$ and leading to $T\left(\mathbb{B}_{k}^{\prime}\right)$, i.e. defined by the equality

$$
T\left(\mathbb{B}_{k}^{\prime}, \mathbb{B}_{j}\right) T\left(\mathbb{B}_{j}\right)=T\left(\mathbb{B}_{k}^{\prime}\right) .
$$

We have obtained a representation of the transformation $T\left(\mathbb{B}_{k}^{\prime}, \mathbb{B}_{j}\right)$, and we formulate the above results in the lemma

Lemma 4.1. The transformation $T\left(\mathbb{B}_{k}^{\prime}, \mathbb{B}_{j}\right)$ has the following Gaussian kernel:

const. $\exp \left[-\frac{1}{2} \beta L^{k(d-2)}\left\langle\psi^{\prime}-Q\left(\mathbb{B}_{k}^{\prime}, \mathbb{B}_{j}\right) \psi, a\left(\mathbb{B}_{k}^{\prime}, \mathbb{B}_{j}\right)\left(\psi^{\prime}-Q\left(\mathbb{B}_{k}^{\prime}, \mathbb{B}_{j}\right) \psi\right)\right\rangle\right]$,

where $\psi$ is defined on $\mathbb{B}_{j} \cup \Lambda_{0}, \psi^{\prime}$ is defined on $\mathbb{B}_{k}^{\prime} \cup \Lambda_{0}^{\prime}$, and $\psi=\psi^{\prime}$ on $\bigcup_{p=0}^{j}\left(\Lambda_{p}^{\prime} \cap\right.$ $\left.\Lambda_{p}\right)$. The scalar product above is defined for the $\eta$-scale, i.e. the sum over points of $\Lambda_{p}^{\prime} \cap \Omega_{p}^{(p) c}$ is multiplied by the volume $\left(L^{p} \eta\right)^{d}$. The constant in front of the exponential is the normalization constant. 
The generalization of the variational problem (2.6) in [1] can be obtained by applying the saddle point method to the integral in

$$
T\left(\mathbb{B}_{k}^{\prime}, \mathbb{B}_{j}\right) \exp \left[-\beta L^{j(d-2)} A\left(\mathbb{B}_{j} ; \psi, \phi\left(\mathbb{B}_{j} ; \psi\right) ; h, a\left(\mathbb{B}_{j}\right), \lambda_{j}, v_{j}\right)\right] .
$$

This leads to the variational problem

$$
\begin{aligned}
\inf _{\psi}\{ & \frac{1}{2}\left\langle\psi^{\prime}-Q\left(\mathbb{B}_{k}^{\prime}, \mathbb{B}_{j}\right) \psi, a\left(\mathbb{B}_{k}^{\prime}, \mathbb{B}_{j}\right)\left(\psi^{\prime}-Q\left(\mathbb{B}_{k}^{\prime}, \mathbb{B}_{j}\right) \psi\right)\right\rangle \\
& \left.\quad+A^{\eta}\left(\mathbb{B}_{j} ; \psi, \phi\left(\mathbb{B}_{j} ; \psi\right) ; h, a\left(\mathbb{B}_{j}\right)\left(L^{j} \eta\right)^{-2}, \lambda_{j}\left(L^{j} \eta\right)^{-2}, v_{j}\left(L^{j} \eta\right)^{-2}\right)\right\},
\end{aligned}
$$

where $\psi^{\prime}$ is a fixed configuration on $\mathbb{B}_{k}^{\prime} \cup \Lambda_{0}^{\prime}, \psi$ is a variable configuration defined on $\mathbb{B}_{j} \cup \Lambda_{0}$ and satisfying the restriction $\psi=\psi^{\prime}$ on $\bigcup_{p=0}^{j}\left(\Lambda_{p}^{\prime} \cap \Lambda_{p}\right)$. The action is rescaled to the $\eta$-lattice, and we denote the rescaled coefficients by $a^{\eta}\left(\mathbb{B}_{j}\right), \lambda_{k}, v_{k}$. For simplicity we drop the superscript " $\eta$ " and the subscript " $k$ " in formulas below. The value of the action in (4.14) is equal to the infimum (1.6), or to (1.9), and the above variational problem can be reformulated as the problem

$$
\begin{aligned}
\inf _{\psi} \inf _{\phi} \sup _{\alpha}\{ & \frac{1}{2}\left\langle\psi^{\prime}-Q\left(\mathbb{B}_{k}^{\prime}, \mathbb{B}_{j}\right) \psi, a\left(\mathbb{B}_{k}^{\prime}, \mathbb{B}_{j}\right)\left(\psi^{\prime}-Q\left(\mathbb{B}_{k}^{\prime}, \mathbb{B}_{j}\right) \psi\right)\right\rangle \\
& +\frac{1}{2}\left\langle\psi-Q\left(\mathbb{B}_{j}\right) \phi, a\left(\mathbb{B}_{j}\right)\left(\psi-Q\left(\mathbb{B}_{j}\right) \phi\right)\right\rangle \\
& \left.+\frac{1}{2}\|\partial \phi\|^{2}+\frac{1}{2}\left\langle\alpha,|\phi|^{2}-1\right\rangle-\frac{1}{2 \lambda}\|\alpha\|^{2}+\frac{v}{2}\|\phi-h\|^{2}\right\},
\end{aligned}
$$

where $\psi^{\prime}$ is a fixed configuration on $\mathbb{B}_{k}^{\prime} \cup \Lambda_{0}^{\prime}$, and $\psi, \phi$ satisfy the restrictions

$$
\begin{gathered}
\psi=\psi^{\prime} \text { on } \bigcup_{p=0}^{J}\left(\Lambda_{p}^{\prime} \cap \Lambda_{p}\right), \phi=\psi \quad \text { on } \Omega_{1}^{c}=\Lambda_{0}, \\
\text { hence } \phi=\psi=\psi^{\prime} \quad \text { on } \Omega_{1}^{\prime c}=\Lambda_{0}^{\prime} .
\end{gathered}
$$

There are some simple relations between the two problems (4.14)-(4.15). A domain of the function of $\psi$ in (4.14) is the set of all $\psi$ such that $(\psi, h) \in \tilde{\Psi}\left(\mathbb{B}_{j} ; c_{1}\right)$. It follows from Proposition 1.1 and the equality (1.8) that if $\tilde{\psi}$ is a critical point of this function, then $\tilde{\psi}, \phi\left(\mathbb{B}_{j} ; \tilde{\psi}\right),\left(L^{j} \eta\right)^{-2} \alpha\left(\mathbb{B}_{j} ; \psi^{\prime}\right)$ is a critical point of the function in the curly brackets in (4.15). Let us study these critical points. The corresponding variational equations are

$$
\chi_{\mathbb{B}_{j}} Q^{*}\left(\mathbb{B}_{k}^{\prime}, \mathbb{B}_{j}\right) a\left(\mathbb{B}_{k}^{\prime}, \mathbb{B}_{j}\right)\left(Q\left(\mathbb{B}_{k}^{\prime}, \mathbb{B}_{j}\right) \psi-\psi^{\prime}\right)+a\left(\mathbb{B}_{J}\right)\left(\psi-Q\left(\mathbb{B}_{j}\right) \phi\right)=0,
$$

or more explicitly, for $y^{\prime} \in \Lambda_{p}^{\prime} \backslash \Lambda_{p}, y \in \Delta_{p}\left(y^{\prime}\right) \cap \Lambda_{n}, n \geqq 1$,

$$
\begin{gathered}
a\left(\Delta_{p}\left(y^{\prime}\right), \mathbb{B}_{j}\right)\left(L^{p} \eta\right)^{-2}\left(Q\left(\Delta_{p}\left(y^{\prime}\right), \mathbb{B}_{j}\right) \psi-\psi_{p}^{\prime}\left(y^{\prime}\right)\right) \\
+a_{n}\left(L^{n} \eta\right)^{-2}\left(\psi_{n}(y)-\left(Q_{n} \phi\right)(y)\right)=0
\end{gathered}
$$




$$
\begin{gathered}
\chi_{\Omega_{1}^{c} \cap \Omega_{1}^{\prime}} Q^{*}\left(\mathbb{B}_{k}^{\prime}, \mathbb{B}_{j}\right) a\left(\mathbb{B}_{k}^{\prime}, \mathbb{B}_{j}\right)\left(Q\left(\mathbb{B}_{k}^{\prime}, \mathbb{B}_{j}\right) \psi-\psi^{\prime}\right)-\Delta_{\Omega_{1}^{c} \cap \Omega_{1}^{\prime}}^{D, \eta} \psi_{0} \\
-\chi_{\partial \Omega_{1}^{c}} \Delta^{\eta} \chi_{\Omega_{1}} \phi-\chi_{\partial \Omega_{1}^{\prime}} \Delta^{\eta} \psi_{0}^{\prime}+\alpha \psi_{0}+v\left(\psi_{0}-h\right)=0 \text { on } \Omega_{1}^{c} \cap \Omega_{1}^{\prime}, \\
\chi_{\Omega_{1}} Q^{*}\left(\mathbb{B}_{j}\right) a\left(\mathbb{B}_{j}\right)\left(Q\left(\mathbb{B}_{j}\right) \phi-\psi\right)-\Delta_{\Omega_{1}}^{D, \eta} \phi-\chi_{\partial \Omega_{1}} \Delta^{\eta} \psi_{0}+\alpha \phi+v(\phi-h)=0 \\
\frac{1}{2}\left(|\phi|^{2}-1\right)-\frac{1}{\lambda} \alpha=0 \quad \text { on } \Omega_{1} \\
\frac{1}{2}\left(\left|\psi_{0}\right|^{2}-1\right)-\frac{1}{\lambda} \alpha=0 \quad \text { on } \Omega_{1}^{c} \cap \Omega_{1}^{\prime} .
\end{gathered}
$$

Writing these equations we have used the restrictions (4.16), or even more precisely $\phi=\psi_{0}$ on $\Omega_{1}^{c} \cap \Omega_{1}^{\prime}, \phi=\psi_{0}=\psi_{0}^{\prime}$ on $\Omega_{1}^{\prime c}$. Notice that the third and fourth equations are exactly Eq. (1.10) for $\mathbb{I}_{j}$, rescaled to the $\eta$-scale. The first equation, or the more explicitly written system of equations, is a very simple system of linear equations for variables $\psi$ on $\mathbb{B}_{J} \backslash \mathbb{B}_{k}^{\prime}$, and it is easy to see that it has a unique solution for all $\psi^{\prime}, \phi$ in corresponding vector spaces. We take the explicitly written equations, divide by $a_{n}\left(L^{n} \eta\right)^{-2}$, multiply by $\left(L^{n} L^{-p}\right)^{d}$, sum over $y \in \Delta_{p}\left(y^{\prime}\right) \cap \Lambda_{n}$, and sum over $n$. We obtain an equation from which we calculate $Q\left(\Delta_{p}\left(y^{\prime}\right), \mathbb{B}_{j}\right) \psi$, substitute it into the original equation and calculate finally the solution $\psi$. It is given by the formula

$$
\begin{aligned}
\psi_{n}(y)= & \left(Q_{n} \phi\right)(y)+\frac{a\left(y^{\prime} ; \mathbb{B}_{k}^{\prime}, \mathbb{B}_{j}\right)}{a\left(y ; \mathbb{B}_{j}\right)}\left[Q\left(\Delta_{p}\left(y^{\prime}\right), \mathbb{B}_{\jmath}\right) \frac{a\left(y^{\prime} ; \mathbb{B}_{k}^{\prime}, \mathbb{B}_{j}\right)}{a\left(\cdot ; \mathbb{B}_{j}\right)}+1\right]^{-1} \\
& \times\left(\psi_{p}^{\prime}\left(y^{\prime}\right)-\left(Q_{p} \phi\right)\left(y^{\prime}\right)\right)
\end{aligned}
$$

where $y \in \Delta_{p}\left(y^{\prime}\right) \cap \Lambda_{n}, y^{\prime} \in \Lambda_{p}^{\prime} \backslash \Lambda_{p}$. It is clear that this solution is also a solution of the following variational problem: find the infimum with respect to $\psi$ of the sum of the first two quadratic forms in (4.15). By the first equality in (4.2), which is valid quite generally, this infimum determines a composition of the two transformations determined by the two quadratic forms. This composition is given by the formula (4.11), so the resulting quadratic form is equal to (1.5), in particular at the point $y^{\prime} \in \Lambda_{p}^{\prime}$ we get the form $\frac{1}{2} \beta L^{p(d-2)} a_{p}\left|\psi_{p}^{\prime}\left(y^{\prime}\right)-\left(Q_{p} \phi\right)\left(y^{\prime}\right)\right|^{2}$. It can be calculated also by substituting the above solution into the sum of the quadratic forms. After simple calculations we obtain a quadratic form, which is a sum over $y^{\prime} \in \mathbb{B}_{k}^{\prime}$ of the forms

$$
\begin{aligned}
\frac{1}{2} \beta L^{p(d-2)} a\left(\Delta_{p}\left(y^{\prime}\right), \mathbb{B}_{j}\right) & {\left[Q\left(\Delta_{p}\left(y^{\prime}\right), \mathbb{B}_{j}\right) \frac{a\left(y^{\prime} ; \mathbb{B}_{k}^{\prime}, \mathbb{B}_{j}\right)}{a\left(\cdot ; \mathbb{B}_{j}\right)}+1\right]^{-1} } \\
\times & \left|\psi_{p}^{\prime}\left(y^{\prime}\right)-\left(Q_{p} \phi\right)\left(y^{\prime}\right)\right|^{2}
\end{aligned}
$$

Of course the two expressions must be equal, so the constant in front of the quadratic expression above is equal to $\frac{1}{2} \beta L^{p(d-2)} a_{p}$. From this we obtain the identity

$$
Q\left(\Delta_{p}\left(y^{\prime}\right), \mathbb{B}_{j}\right) \frac{a\left(y^{\prime} ; \mathbb{B}_{k}^{\prime}, \mathbb{B}_{j}\right)}{a\left(\cdot ; \mathbb{B}_{j}\right)}+1=\frac{a\left(\Delta_{p}\left(y^{\prime}\right), \mathbb{B}_{J}\right)}{a_{p}} .
$$

In the above expressions involving the constants $\frac{1}{a_{n}}$ we have to include also the case $n=0$, and for consistency we have to put $\frac{1}{a_{0}}=0$, or $a_{0}=+\infty$. Using the above 
identity we can write the following simple formula for the solution $\psi$ :

$$
\psi_{n}(y)=\left(Q_{n} \phi\right)(y)+\frac{a_{p}}{a_{n}}\left(L^{n} L^{-p}\right)^{2}\left(\psi_{p}^{\prime}\left(y^{\prime}\right)-\left(Q_{p} \phi\right)\left(y^{\prime}\right)\right)
$$

for $y \in \Delta_{p}\left(y^{\prime}\right) \cap \Lambda_{n}, n \geqq 1, y^{\prime} \in \Lambda_{p}^{\prime} \backslash \Lambda_{p}$. We can drop the restriction $y^{\prime} \notin \Lambda_{p}^{\prime} \cap \Lambda_{p}$, because for $y^{\prime} \in \Lambda_{p}^{\prime} \cap \Lambda_{p}$ we have $y=y^{\prime}, n=p$, and the above formula is reduced to the identity $\psi_{n}(y)=\psi_{p}\left(y^{\prime}\right)=\psi_{p}^{\prime}\left(y^{\prime}\right)$. We can extend it also on the domain $\Omega_{1}^{c} \cap$ $\Omega_{1}^{\prime}$, because then we have $n=0, \frac{1}{a_{0}}=0$, hence the second expression on the righthand side vanishes, and the formula is reduced to the identity $\psi_{0}(y)=\phi(y)=$ $\left(Q_{0} \phi\right)(y)$. Thus (4.19) holds on the whole set $\mathbb{B}_{j} \cup\left(\Omega_{1}^{c} \cap \Omega_{1}^{\prime}\right)$. It can be written also in the following form:

$$
\psi=Q\left(\mathbb{B}_{j}\right) \phi+\frac{1}{a\left(\mathbb{B}_{j}\right)} Q^{*}\left(\mathbb{B}_{k}^{\prime}, \mathbb{B}_{j}\right) a\left(\mathbb{B}_{k}^{\prime}\right)\left(\psi^{\prime}-Q\left(\mathbb{B}_{k}^{\prime}\right) \phi\right) \text { on } \mathbb{B}_{j} \cup\left(\Omega_{1}^{c} \cap \Omega_{1}^{\prime}\right) .
$$

Now we write the second equation in (4.17) with $\phi$ instead of $\psi_{0}$, using the identity $\psi_{0}=\phi$ on $\Omega_{1}^{c} \cap \Omega_{1}^{\prime}$, and we substitute the solution $\psi$ into the second and third equations. After some simple transformations with a use of the identity (4.18), and combining the two equations we obtain the first equation in (1.10) for the generating set $\mathbb{B}_{k}^{\prime}$. Combining the fourth and fifth equations in (4.17) we obtain the second equation in (1.10). These statements are very easy to understand if we substitute the solution $\psi$ into the function in (4.15). The sum of the first two quadratic forms is then equal to the form (1.6) for $\mathbb{B}_{k}^{\prime}$, by the composition formula (4.11), and the function in (4.15) is equal to the function (1.7) for $\mathbb{B}_{k}^{\prime}$. The variational equations are then Eq. (1.10) for $\mathbb{B}_{k}^{\prime}$. The above analysis and Proposition 1.1 lead to conclusions formulated in the lemma below.

Lemma 4.2. If $\left(\psi^{\prime}, h\right) \in \tilde{\Psi}\left(\mathbb{B}_{k}^{\prime} ; \delta\right), \delta \leqq c_{1}$, then the system of equations (4.17) has a unique solution in the space of all configurations $\psi, \phi, \alpha$ satisfying the restrictions (4.16) and $|\alpha|_{2}^{\prime}<c_{0}$, where $|\alpha|_{2}^{\prime}$ is the norm (3.17) determined by the generating set $\mathbb{B}_{k}^{\prime}$. The configurations $\phi, \alpha$ of this solution satisfy the system of equations (1.10) for the set $\mathbb{B}_{k}^{\prime}$, hence they are equal to $\phi\left(\mathbb{B}_{k}^{\prime} ; \psi^{\prime}\right), \alpha\left(\mathbb{B}_{k}^{\prime} ; \psi^{\prime}\right)$. The configuration $\psi$ of the solution is given then by the formula (4.20), so it is equal to the function

$$
\begin{aligned}
\psi\left(\mathbb{B}_{j}, \mathbb{B}_{k}^{\prime} ; \psi^{\prime}\right)= & Q\left(\mathbb{B}_{j}\right) \phi\left(\mathbb{B}_{k}^{\prime} ; \psi^{\prime}\right) \\
& +\frac{1}{a\left(\mathbb{B}_{j}\right)} Q^{*}\left(\mathbb{B}_{k}^{\prime}, \mathbb{B}_{j}\right) a\left(\mathbb{B}_{k}^{\prime}\right)\left(\psi^{\prime}-Q\left(\mathbb{B}_{k}^{\prime}\right) \phi\left(\mathbb{B}_{k}^{\prime} ; \psi^{\prime}\right)\right),
\end{aligned}
$$

defined on $\mathbb{B}_{J} \cup\left(\Omega_{1}^{c} \cap \Omega_{1}^{\prime}\right)$. The functions determining the solution have the analytic extensions onto the spaces $\tilde{\Psi}^{c}\left(\mathbb{B}_{k}^{\prime} ; \delta, \varepsilon\right)$ with $\delta, \varepsilon$ satisfying the assumptions of Proposition 1.1, and they satisfy also the naturally extended equations (4.17), i.e. like Eq. (1.14), with $\psi^{\prime}$, h replaced by the corresponding complex configurations.

This lemma answers completely the questions about critical points of the function in (4.15). There exists exactly one critical point in the domain $\left\{|\alpha|_{2}^{\prime}<c_{0}\right\}$, the solution of the system (4.17), and it is also easy to see that it is a solution of the variational problem (4.15) by expanding the function around the critical point and considering second order terms of the expansion, as in (3.48). This is of no particular importance for us, so we omit this simple calculation. 
Our fundamental goal is to understand the variational problem (4.14), or more generally critical points of the function in (4.14). We have noticed already that if $\tilde{\psi}$ is such a critical point, then $\tilde{\psi}, \phi\left(\mathbb{B}_{j} ; \tilde{\psi}\right),\left(L^{j} \eta\right)^{-2} \alpha\left(\mathbb{B}_{j} ; \tilde{\psi}\right)$ is a solution of the system (4.17). We have constructed the solution $\psi\left(\mathbb{B}_{j}, \mathbb{B}_{k}^{\prime}\right), \phi\left(\mathbb{B}_{k}^{\prime}\right), \alpha\left(\mathbb{B}_{k}^{\prime}\right)$ of this system, so the question is if $\psi\left(\mathbb{B}_{j}, \mathbb{B}_{k}^{\prime}\right)$ is a critical point of the function in (4.14). Quite generally, let us take a solution $\tilde{\psi}, \tilde{\phi}, \tilde{\alpha}$ of the system (4.17), always assuming the restrictions (4.16). Consider the third and fourth equations in this system. Rescaling them to the $L^{-J}$-lattice we obtain that $\tilde{\psi}, \tilde{\phi},\left(L^{J} \eta\right)^{2} \tilde{\alpha}$ satisfy the system $(1.10)$ for $\mathbb{B}_{j}$ instead of $\mathbb{B}_{k}$. If $(\tilde{\psi}, h) \in \tilde{\Psi}\left(\mathbb{B}_{j} ; c_{1}\right)$ and $\left|\left(L^{j} \eta\right)^{2} \tilde{\alpha}\right|^{2}<c_{0}$, the norm is determined by $\mathbb{B}_{j}$, then the configurations $\tilde{\psi}, \tilde{\phi},\left(L^{j} \eta\right)^{2} \tilde{\alpha}$ restricted to $\Omega_{1}$ yield the unique solution of the system in the domain $\left\{|\alpha|_{2}<c_{0}\right\}$, therefore we obtain, after rescaling them back to the $\eta$-lattice, the identities

$$
\tilde{\phi}=\phi\left(\mathbb{B}_{J} ; \tilde{\psi}\right), \tilde{\alpha}=\left(L^{j} \eta\right)^{-2} \alpha\left(\mathbb{B}_{j} ; \tilde{\psi}\right) \quad \text { on } \Omega_{1} \text {. }
$$

From this we conclude further, taking into account the remaining equations in (4.17), that $\tilde{\psi}$ is a critical point of the function in (4.14). Thus we have to check when $\psi\left(\mathbb{B}_{j}, \mathbb{B}_{k}^{\prime}\right),\left(L^{J} \eta\right)^{2} \alpha\left(\mathbb{B}_{k}^{\prime}\right)$ satisfy the above conditions, i.e. for what $\delta$

$$
\left(\psi\left(\mathbb{B}_{j} ; \mathbb{B}_{k}^{\prime}\right), h\right) \in \tilde{\Psi}\left(\mathbb{B}_{j} ; c_{1}\right),\left|\left(L^{j} \eta\right)^{2} \alpha\left(\mathbb{B}_{k}^{\prime}\right)\right|_{2}<c_{0} .
$$

For simplicity let us denote the solution of the system (4.17) described in Lemma 4.2 by $\psi_{k}^{(j)}, \phi_{k}, \alpha_{k}$. The configurations $\phi_{k}, \alpha_{k}$ satisfy the conditions $(1.15),(1.16)$ of Proposition 1.1, and $\psi_{k}^{(j)}$ is given by the formula (4.21). Take a bond $\left\langle y_{1}, y_{2}\right\rangle$ $\subset \Lambda_{n}$ and denote by $y_{1}^{\prime}, y_{2}^{\prime}$ the points of $\mathbb{B}_{k}^{\prime}$ such that $y_{1}^{\prime} \in \Lambda_{p_{1}}^{\prime}, y_{2}^{\prime} \in \Lambda_{p_{2}}^{\prime}$, $y_{1} \in \Delta_{p_{1}}\left(y_{1}^{\prime}\right), y_{2} \in \Delta_{p_{2}}\left(y_{2}^{\prime}\right)$. Of course $p_{1} \geqq n, p_{2} \geqq n,\left|p_{1}-p_{2}\right| \leqq 1$, and we have

$$
\begin{gathered}
\left|\psi_{k}^{(j)}\left(y_{1}\right)-\psi_{k}^{(j)}\left(y_{2}\right)\right| \leqq\left|\left(Q_{n} \phi_{k}\right)\left(y_{1}\right)-\left(Q_{n} \phi_{k}\right)\left(y_{2}\right)\right| \\
+\frac{a_{p_{1}}}{a_{n}}\left(L^{n} L^{-p_{1}}\right)^{2}\left|\psi_{p_{1}}^{\prime}\left(y_{1}^{\prime}\right)-\left(Q_{p_{1}} \phi_{k}\right)\left(y_{1}^{\prime}\right)\right| \\
+\frac{a_{p_{2}}}{a_{n}}\left(L^{n} L^{-p_{2}}\right)^{2}\left|\psi_{p_{2}}^{\prime}\left(y_{2}^{\prime}\right)-\left(Q_{p_{2}} \phi_{k}\right)\left(y_{2}^{\prime}\right)\right|<L^{n} L^{-\min \left\{p_{1}, p_{2}\right\}} K_{1} \delta \\
+\left(L^{n} L^{-p_{1}}\right)^{2} K_{1} \delta+\left(L^{n} L^{-p_{2}}\right)^{2} K_{1} \delta \leqq 3 K_{1} \delta .
\end{gathered}
$$

If the bond $\left\langle y_{1}, y_{2}\right\rangle \subset T^{(n)}$ is such that $y_{1} \in \Lambda_{n-1}, y_{2} \in \Lambda_{n}$, and $x \in B\left(y_{1}\right)$, then the only important difference in the above bound is that we have the term $\left|\left(Q_{n-1} \phi_{k}\right)(x)-\left(Q_{n} \phi_{k}\right)\left(y_{2}\right)\right|$ on the right-hand side, and it can be bounded by $d K_{1} \delta$, so the overall bound is $(d+2) K_{1} \delta$. Take now a point $y \in \Lambda_{n}$ and denote again by $y^{\prime}$ the point of $\mathbb{B}_{k}^{\prime}$ such that $y^{\prime} \in \Lambda_{p}^{\prime}, y \in \Delta_{p}\left(y^{\prime}\right)$. Then $p \geqq n$ and we have

$$
\begin{aligned}
& \left\|\psi_{k}^{(j)}(y)|-1|<\right\|\left(Q_{n} \phi_{k}\right)(y)|-1|+\left(L^{n} L^{-p}\right)^{2} K_{1} \delta \\
& \leqq \|\left.\left(Q_{n} \phi_{k}\right)(y)\right|^{2}-1\left|+K_{1} \delta \leqq \frac{1}{2} \sum_{x_{1}, x_{2} \in B_{n}(y)} L^{-2 n d}\right| \phi_{k}\left(x_{1}\right)-\left.\phi_{k}\left(x_{2}\right)\right|^{2} \\
& \quad+\left(Q_{n} \|\left.\phi_{k}\right|^{2}-1 \mid\right)(y)+K_{1} \delta<\frac{1}{2} d^{2}\left(L^{n} L^{-p}\right)^{2} K_{1}^{2} \delta^{2}+\frac{2}{\lambda}\left(Q_{n}\left|\alpha_{k}\right|\right)(y)+K_{1} \delta \\
& \quad<\frac{1}{2} d^{2} K_{1}^{2} \delta^{2}+\frac{2}{\lambda}\left(L^{p} \eta\right)^{-2} K_{1} \delta+K_{1} \delta<2 K_{1} \delta
\end{aligned}
$$


where we have used the assumptions $\lambda \eta^{2} \geqq 1, L \geqq 3$, and $\delta$ sufficiently small, e.g. $d^{2} K_{1} \delta \leqq 1$. Further, we have

$$
\begin{aligned}
\mid \psi_{k}^{(j)}(y)- & h \mid<\left(Q_{n}\left|\phi_{k}-h\right|\right)(y)+K_{1} \delta<\left(L^{p} \eta\right)^{-1} K_{1} \frac{\delta}{\sqrt{v}}+K_{1} \delta \\
& \leqq 2\left(L^{p} \eta\right)^{-1} K_{1} \frac{\delta}{\sqrt{v_{k}}}=2 L^{j} L^{-p} K_{1} \frac{\delta}{\sqrt{v_{j}}} \leqq 2\left(L^{n} \xi\right)^{-1} K_{1} \frac{\delta}{\sqrt{v_{j}}}
\end{aligned}
$$

where $\xi=L^{-j}$ and $v_{j}=\left(L^{j} \eta\right)^{2} v_{k}=\left(L^{j} \eta\right)^{2} v$ is the rescaled constant $v$. From the above inequality we obtain

$$
\begin{aligned}
& \left(\left|\psi_{k}^{(j)}(y)\right|-1\right)^{2}+\left(|h|^{2}-1\right)+2\left(\left|\psi_{k}^{(j)}(y)\right|-\psi_{k}^{(J)}(y) \cdot h\right) \\
& =\left|\psi_{k}^{(J)}(y)-h\right|^{2}<4\left(L^{n} \xi\right)^{-2} K_{1} \frac{2 \delta^{2}}{v_{j}}
\end{aligned}
$$

hence using the previous bound on $\left|\psi_{k}^{(j)}(y)\right|-1$ and the condition on $h$ in the definition of $\tilde{\Psi}\left(\mathbb{B}_{k}^{\prime} ; \delta\right)$ we obtain

$$
\left|\psi_{k}^{(j)}(y)\right|-\psi_{k}^{(j)}(y) \cdot h<2\left(L^{n} \xi\right)^{-2} K_{1}^{2} \frac{\delta^{2}}{v_{j}}+2 K_{1}^{2} \delta^{2}+\frac{1}{2} \frac{\delta^{2}}{v_{k}}<5\left(L^{n} \xi\right)^{-2} K_{1}^{2} \frac{\delta^{2}}{v_{j}}
$$

and

$$
v_{j}\left(1-\left(\psi_{k}^{(j)}(y)\right)_{0} \cdot h\right)<\frac{5}{\left|\psi_{k}^{(j)}(y)\right|}\left(L^{n} \xi\right)^{-2} K_{1}^{2} \delta^{2}<10\left(L^{n} \xi\right)^{-2} K_{1}^{2} \delta^{2} .
$$

The above inequalities hold also for $n=0$. Let us make a few comments on these inequalities. Basically we have repeated the arguments (3.1)-(3.6) [1] of the proof of Lemma 3.1 in [1], only taking into account various scaling factors $L^{n} L^{-p}$. Actually we can improve them keeping track of those scaling factors more carefully, but we do not need such improved inequalities, except the case when we consider them on a domain $X \subset \Omega_{k}^{\prime} \cap \Omega_{j}$. Then we get additional scaling factors which are the same powers of $L^{j} \eta$ as the powers of $\sigma$ in the definition (3.13) [1] of the spaces $\Psi_{j}(\sigma, \varepsilon)$. We introduce localized spaces $\Psi_{j}(X ; \sigma, \varepsilon)$ by the same definition (3.13) [1], but with all conditions restricted to a domain $X$. Then the above improved inequalities can be summarized as the statement that the configuration $\left(\psi_{k}^{(J)}, h\right)$ restricted to $X$ belongs to the space $\Psi_{j}\left(X ; 3 L^{j} \eta, K_{1} \delta\right)$. In this case we improve also the constant replacing $d+2$ by 3 , as in Lemma 3.1 [1]. Notice further that in $(4.24)-(4.27)$ we have used only the fact that $\psi_{k}^{(j)}$ is related to $\phi_{k}$ by the inequality

$$
\left|\psi_{k}^{(j)}(y)-\left(Q_{n} \phi_{k}\right)(y)\right|<\left(L^{n} L^{-p}\right)^{2}\left|\psi^{\prime}\left(y^{\prime}\right)-\left(Q_{p} \phi_{k}\right)\left(y^{\prime}\right)\right|<\left(L^{n} L^{-p}\right)^{2} K_{1} \delta,
$$

where $y \in \Lambda_{n}, y \in \Delta_{p}\left(y^{\prime}\right), y^{\prime} \in \Lambda_{p}^{\prime}$, and that $\phi_{k}, \alpha_{k}$ satisfy the conditions (1.15), (1.16), and the second equation in (1.10) or (1.14). From this we obtain a generalization of Lemma 3.1 in [1] which will be formulated later on. Let us formulate a part of these conclusions in the following lemma. 
Lemma 4.3. If $\delta \leqq c_{1},\left(\psi^{\prime}, h\right) \in \tilde{\Psi}\left(\mathbb{B}_{k}^{\prime} ; \delta\right), X \subset \Omega_{k}^{\prime} \cap \Omega_{j}$, then

$$
\begin{aligned}
& \left(\psi\left(\mathbb{B}_{j}, \mathbb{B}_{k}^{\prime} ; \psi^{\prime}\right), h\right) \in \tilde{\Psi}\left(\mathbb{B}_{j} ;(d+2) K_{1} \delta\right), \\
& \left(\psi\left(\mathbb{B}_{j}, \mathbb{B}_{k}^{\prime} ; \psi^{\prime}\right), h\right)\left\lceil_{X} \in \Psi_{j}\left(X ; 3 L^{j} \eta, K_{1} \delta\right) .\right.
\end{aligned}
$$

Finally, for $x \in B_{n}(y)$ we have

$$
\left(L^{n} \xi\right)^{2}\left|\left(L^{j} \eta\right)^{2} \alpha_{k}(x)\right|=\left(L^{n} \eta\right)^{2}\left|\alpha_{k}(x)\right| \leqq\left(L^{p} \eta\right)^{2}\left|\alpha_{k}(x)\right|<K_{1} \delta \leqq K_{1} c_{1} \leqq c_{0} .
$$

From (4.29), (4.31) we obtain that the conditions (4.23) are satisfied if $(d+2)$ $K_{1} \delta \leqq c_{1}$. Assuming this we obtain the identities

$$
\phi_{k}=\phi\left(\mathbb{B}_{j} ; \psi_{k}^{(j)}\right), \quad \alpha_{k}=\left(L^{j} \eta\right)^{-2} \alpha\left(\mathbb{B}_{j} ; \psi_{k}^{(j)}\right) .
$$

From these identities we conclude also that $\psi_{k}^{(j)}$ is a critical point of the function (4.14). Unfortunately we cannot conclude that it is a unique critical point of this function considered on the domain $\tilde{\Psi}\left(\mathbb{B}_{j} ; c_{1}\right)$. To obtain uniqueness we have to restrict this space. Let us recall again that if $\psi$ is a critical point, then $\phi\left(\mathbb{B}_{j} ; \tilde{\psi}\right),\left(L^{n} \eta\right)^{-2} \alpha\left(\mathbb{B}_{j} ; \tilde{\psi}\right)$ is a solution of the system $(1.10)$ for the set $\mathbb{B}_{k}^{\prime}$ and the configuration $\psi^{\prime}$. If $\left(\psi^{\prime}, h\right) \in \tilde{\Psi}\left(\mathbb{B}_{k}^{\prime} ; c_{1}\right)$ and $\left|\left(L^{j} \eta\right)^{-2} \alpha\left(\mathbb{B}_{j} ; \tilde{\psi}\right)\right|_{2}^{\prime}<c_{0}$, then this solution is unique and equal to $\phi_{k}, \alpha_{k}$. Thus we have the uniqueness in the domain $\tilde{\Psi}\left(\mathbb{B}_{j} ; c_{1}\right)$ restricted by the condition $\left|\left(L^{j} \eta\right)^{-2} \alpha\left(\mathbb{B}_{j} ; \psi\right)\right|_{2}^{\prime}<c_{0}$.

We have finished the discussion of the variational problems (4.14), (4.15) and the related equations (4.17). Let us stress that the most important result is the construction of the function $\psi\left(\mathbb{B}_{j}, \mathbb{B}_{k}^{\prime}\right)$ satisfying the identities (4.32). These identities play a crucial role in our renormalization group approach, and we need them also for the analytically extended functions. They are not immediate because the analytic extensions are not uniquely defined, and we have to refer again to the analytically extended equations (4.17). To repeat the above arguments leading to the identities (4.32) we have to prove a statement corresponding to (4.29) for the complex spaces. Take a space $\tilde{\Psi}^{c}\left(\mathbb{B}_{k}^{\prime} ; \delta, \varepsilon\right)$ with $\delta, \varepsilon$ satisfying the assumptions of Proposition 1.1 . We use the same simplified notations for the considered functions as before. The functions $\phi_{k}, \alpha_{k}$ have the analytic extensions onto this space, therfore the function $\psi_{k}^{(j)}$ has also the extension defined by the formula (4.21). Denote elements of the space by $\left(\psi^{\prime}+\delta \psi^{\prime}, h+h^{\prime}\right)$, where $\left(\psi^{\prime}, h\right)$ are elements of the real subspace $\tilde{\Psi}\left(\mathbb{B}_{k}^{\prime} ; \delta\right)$, and write the complex functions in the form $\phi_{k}+\delta \phi_{k}, \alpha_{k}+\delta \alpha_{k}, \psi_{k}^{(j)}+\delta \psi_{k}^{(j)}$, where $\phi_{k}, \alpha_{k}, \psi_{k}^{(j)}$ are the corresponding real functions, as in the last formula in (1.15). Notice that $\delta \psi_{k}^{(j)}$ has the representation (4.21), but with $\phi_{k}, \psi^{\prime}$ replaced by $\delta \phi_{k}, \delta \psi^{\prime}$. The bounds (1.15) hold for the complex functions, hence we can bound the differences $\delta \psi_{k}^{(j)}\left(y_{1}\right)-\delta \psi_{k}^{(j)}\left(y_{2}\right)$ as before, but we obtain the constant $2(d+2) K_{1} \delta$ on the right-hand side. Further we have

$$
\begin{aligned}
\left|\delta \psi_{k}^{(j)}(y)\right| \leqq & \left(Q_{n}\left|\delta \phi_{k}\right|\right)(y)+\frac{a_{p}}{a_{n}}\left(L^{n} L^{-p}\right)^{2}\left|\delta \psi^{\prime}\left(y^{\prime}\right)-\left(Q_{p} \delta \phi_{k}\right)\left(y^{\prime}\right)\right| \\
< & \varepsilon+K_{1} \delta+\left|\left(\psi^{\prime}\left(y^{\prime}\right)+\delta \psi^{\prime}\left(y^{\prime}\right)\right)-\left(Q_{p}\left(\phi_{k}+\delta \phi_{k}\right)\right)\left(y^{\prime}\right)\right| \\
& +\left|\psi^{\prime}\left(y^{\prime}\right)-\left(Q_{p} \phi_{k}\right)\left(y^{\prime}\right)\right|<\varepsilon+3 K_{1} \delta
\end{aligned}
$$


and

$$
\begin{gathered}
\left|\left(\psi_{k}^{(j)}(y)+\delta \psi_{k}^{(j)}(y)\right)^{2}-\left(\psi_{k}^{(j)}(y)\right)^{2}\right|<\left|\left(Q_{n}\left(\phi_{k}+\delta \phi_{k}\right)\right)^{2}(y)-\left(Q_{n} \phi_{k}\right)^{2}(y)\right| \\
+2\left(3 K_{1} \delta+K_{1}^{2} \delta^{2}\right) \leqq \frac{1}{2} \sum_{x, x^{\prime} \in B_{n}(y)} L^{-2 n d}\left|\left(\phi_{k}+\delta \phi_{k}\right)(x)-\left(\phi_{k}+\delta \phi_{k}\right)\left(x^{\prime}\right)\right|^{2} \\
+\frac{1}{2} \sum_{x, x^{\prime} \in B_{n}(y)} L^{-2 n d}\left|\phi_{k}(x)-\phi_{k}\left(x^{\prime}\right)\right|^{2}+\left(Q_{n}\left|\left(\phi_{k}+\delta \phi_{k}\right)^{2}-\phi_{k}^{2}\right|\right)(y)+6 K_{1} \delta+2 K_{1}^{2} \delta^{2} \\
<d^{2}\left(L^{n} L^{-p}\right)^{2} K_{1}^{2} \delta^{2}+\frac{2}{\lambda}\left(Q_{n} \mid\left(\delta \alpha_{k} \mid\right)(y)+6 K_{1} \delta+2 K_{1}^{2} \delta^{2}<6 K_{1} \delta+\left(d^{2}+2\right) K_{1}^{2} \delta^{2}\right. \\
+\frac{2}{\lambda}\left(L^{p} \eta\right)^{-2} K_{1} \delta<9 K_{1} \delta, \text { where } y \in \Delta_{p}\left(y^{\prime}\right) \cap \Lambda_{n}, y^{\prime} \in \Lambda_{p}^{\prime},
\end{gathered}
$$

and we have used again the assumptions $\lambda \eta^{2} \geqq 1, L \geqq 3, d^{2} K_{1} \delta \leqq 1$. Take a configuration $h^{\prime}$ satisfying the last two conditions in the definition (1.12) of the space $\tilde{\Psi}^{c}\left(\mathbb{B}_{k}^{\prime} ; \delta, \varepsilon\right)$. It is defined on $\mathbb{B}_{k}^{\prime}$ and $\left|v_{k} h^{\prime}\left(y^{\prime}\right)\right|<\left(L^{p} \eta\right)^{-2} \frac{\delta^{2}}{\varepsilon}$ for $y^{\prime} \in \Lambda_{p}^{\prime}$, hence for $y \in \Delta_{p}\left(y^{\prime}\right) \cap \Lambda_{n}$ we have

$$
\left|v_{j}\left(Q^{*}\left(\mathbb{B}_{k}^{\prime}, \mathbb{B}_{j}\right) h^{\prime}\right)(y)\right|=\left(L^{j} \eta\right)^{2}\left|v_{k} h^{\prime}\left(y^{\prime}\right)\right|<\left(L^{p} L^{-j}\right)^{-2} \frac{\delta^{2}}{\varepsilon} \leqq\left(L^{n} \xi\right)^{-2} \frac{\delta^{2}}{\varepsilon},
$$

and the configuration $Q^{*}\left(\mathbb{B}_{k}^{\prime}, \mathbb{B}_{j}\right) h^{\prime}$ satisfies the first of the two conditions for the space $\tilde{\Psi}^{c}\left(\mathbb{B}_{j} ; \delta, \varepsilon\right)$. To check the second condition we estimate the product

$$
\begin{aligned}
& \left|v_{j}\left(\psi_{k}^{(j)}(y)\right)_{0}\left(Q^{*}\left(\mathbb{B}_{k}^{\prime}, \mathbb{B}_{j}\right) h^{\prime}\right)(y)\right|=\frac{\left(L^{j} \eta\right)^{2}}{\left|\psi_{k}^{(j)}(y)\right|}\left|v_{k} \psi_{k}^{(j)}(y) \cdot h^{\prime}\left(y^{\prime}\right)\right| \\
& <2\left(L^{j} \eta\right)^{2}\left(Q_{n}\left|v_{k} \phi_{k} \cdot h^{\prime}\left(y^{\prime}\right)\right|\right)(y)+2\left(L^{n} L^{-p}\right)^{2} K_{1} \delta\left(L^{p} L^{-j}\right)^{-2} \frac{\delta^{2}}{\varepsilon} \\
& <2\left(L^{j} \eta\right)^{2}\left(L^{p} \eta\right)^{-2} K_{1} \delta^{2}+2\left(L^{n} \xi\right)^{-2} K_{1} \delta^{2} \leqq\left(L^{n} \xi\right)^{-2} 4 K_{1} \delta^{2} .
\end{aligned}
$$

The above inequalities combined with Lemma 4.3 yield the following lemma:

Lemma 4.4. If $\delta$, $\varepsilon$ satisfy the assumptions of Proposition $1.1,\left(\psi^{\prime}+\delta \psi^{\prime}, h+h^{\prime}\right) \in$ $\tilde{\Psi}^{c}\left(\mathbb{B}_{k}^{\prime} ; \delta, \varepsilon\right)$ and $X \subset \Omega_{k}^{\prime} \cap \Omega_{j}$, then

$$
\begin{gathered}
\left(\psi\left(\mathbb{B}_{j}, \mathbb{B}_{k}^{\prime} ; \psi^{\prime}+\delta \psi^{\prime}\right), h+Q^{*}\left(\mathbb{B}_{k}^{\prime}, \mathbb{B}_{j}\right) h^{\prime}\right) \in \tilde{\Psi}^{c}\left(\mathbb{B}_{j} ; 2(d+2) K_{1} \delta, \varepsilon\right), \\
\left(\psi\left(\mathbb{B}_{j}, \mathbb{B}_{k}^{\prime} ; \psi^{\prime}+\delta \psi^{\prime}\right), h+Q_{k-j}^{*} h^{\prime}\right)\left\lceil_{X} \in \Psi_{j}^{c}\left(X ; 3 L^{j} \eta, K_{1} \delta\right) .\right.
\end{gathered}
$$

Finally, notice that the estimates (4.31) hold also for the complex configuration. Now the rest of the argument goes on like in the real case, the only change is that we consider the complex solutions of the extended system (4.17), i.e. the system in which $\psi^{\prime}, h$ are replaced by $\psi^{\prime}+\delta \psi^{\prime}, h+h^{\prime}$, and we have to assume that $2(d+2) K_{1} \delta, \varepsilon$ satisfy the assumptions of Proposition 1.1 , which reduces to the additional conditions $2(d+2) K_{1} \delta \leqq \varepsilon, 2(d+2) K_{1} \delta \leqq c_{1}$. We may also replace $\varepsilon$ by $2(d+2) K_{1} \varepsilon$ in (4.37), and then the assumptions take the form $2(d+2) K_{1} \varepsilon \leqq$ $c_{0}, 2(d+2) K_{1} \delta \leqq c_{1}$. With these assumptions we obtain the identities (4.32) for the analytically extended functions. 
We summarize the main results in the proposition below.

Proposition 4.1. If $\left(\psi^{\prime}, h\right) \in \tilde{\Psi}\left(\mathbb{B}_{k}^{\prime} ; \delta\right), \delta \leqq c_{1}$, then the function in (4.15) has a unique critical point in the domain of all configurations $\psi, \phi, \alpha$ satisfying the restriction $|\alpha|_{2}^{\prime}<c_{0}$, or equivalently the system of equations (4.17) has a unique solution in this domain. This solution is given by the functions $\psi\left(\mathbb{B}_{j}, \mathbb{B}_{k}^{\prime} ; \psi^{\prime}\right), \phi\left(\mathbb{B}_{k}^{\prime}\right.$; $\left.\psi^{\prime}\right), \alpha\left(\mathbb{B}_{k}^{\prime}, \psi^{\prime}\right)$, where the first function is given by the formula (4.21) and satisfies (4.29). If $(d+2) K_{1} \delta \leqq c_{1}$, then $\psi\left(\mathbb{B}_{j}, \mathbb{B}_{k}^{\prime} ; \psi^{\prime}\right)$ is a critical point of the function in (4.14), actually it is a solution of the variational problem (4.14), and it is a unique critical point in the domain $\left\{\psi:(\psi, h) \in \tilde{\Psi}\left(\mathbb{B}_{j} ; c_{1}\right),\left|\left(L^{j} \eta\right)^{-2} \alpha\left(\mathbb{B}_{j} ; \psi\right)\right|_{2}^{\prime}<c_{0}\right\}$. The function has analytic extensions onto domains $\tilde{\Psi}^{c}\left(\mathbb{B}_{k}^{\prime} ; \delta, \varepsilon\right)$ with $\delta, \varepsilon$ satisfying the assumptions of Proposition 1.1, the extensions determined by the formula (4.21) and by Proposition 1.1, and satisfying (4.37). If $4 d K_{1} \delta \leqq \varepsilon, 4 d K_{1} \delta \leqq c_{1}$, or if $4 d K_{1} \varepsilon \leqq c_{0}, 4 d K_{1} \delta \leqq c_{1}$, then these analytic extensions satisfy the identities

$\phi\left(\mathbb{B}_{j} ; \psi\left(\mathbb{B}_{j}, \mathbb{B}_{k}^{\prime} ; \psi^{\prime}\right)\right)=\phi\left(\mathbb{B}_{k}^{\prime} ; \psi^{\prime}\right),\left(L^{j} \eta\right)^{-2} \alpha\left(\mathbb{B}_{j} ; \psi\left(\mathbb{B}_{j}, \mathbb{B}_{k}^{\prime} ; \psi^{\prime}\right)\right)=\alpha\left(\mathbb{B}_{k}^{\prime}, \psi^{\prime}\right)$,

where we have assumed that the functions $\phi\left(\mathbb{B}_{j} ; \psi\right), \alpha\left(\mathbb{B}_{j} ; \psi\right)$ are extended beyond $\Omega_{1}$ by putting

$$
\phi\left(x ; \mathbb{B}_{j} ; \psi\right)=\psi(x), \alpha\left(x ; \mathbb{B}_{j} ; \psi\right)=\frac{\lambda_{j}}{2}\left(\psi^{2}(x)-1\right) \text { for } x \in \Omega_{1}^{c},
$$

and similarly the functions $\phi\left(\mathbb{B}_{k}^{\prime} ; \psi^{\prime}\right), \alpha\left(\mathbb{B}_{k}^{\prime} ; \psi^{\prime}\right)$ beyond $\Omega_{1}^{\prime}$.

Let us mention now some applications of the functions $\psi\left(\mathbb{B}_{j}, \mathbb{B}_{k}^{\prime}\right)$ constructed above. One application was described in the paper [1], where the inductive assumptions on the effective actions were formulated in terms of the functions $\psi_{k}^{(j)}$. In dealing with the "large field" problem we will use more general $\psi\left(\mathbb{B}_{j}, \mathbb{B}_{k}^{\prime}\right)$, for which $\Omega_{n}^{\prime}=\Omega_{n}$ for $n \leqq j$. The generating sets $\mathbb{B}_{k}^{\prime}$ will be used also as determining a kind of "soft" boundary conditions, and in such role these functions will be used in full generality in various localization problems, i.e. in constructing expansions of the type described in the inductive hypothesis (H.5) in [1].

We will need also the functions and the composition formula (4.39) in the case when the basic system of equations is the system (3.20). It corresponds to the variational problem

$$
\begin{aligned}
& \inf _{\delta \psi} \inf _{\delta \phi} \sup _{\delta \alpha}\left\{\frac{1}{2}\left\langle\psi^{\prime}-Q\left(\mathbb{B}_{k}^{\prime}, \mathbb{B}_{j}\right) \delta \psi, a\left(\mathbb{B}_{k}^{\prime}, \mathbb{B}_{j}\right)\left(\psi^{\prime}-Q\left(\mathbb{B}_{k}^{\prime}, \mathbb{B}_{j}\right) \delta \psi\right)\right\rangle\right. \\
& +\left[\frac{1}{2}\left\langle\delta \psi-Q\left(\mathbb{B}_{j}\right) \delta \phi, a\left(\mathbb{B}_{j}\right)\left(\delta \psi-Q\left(\mathbb{B}_{j}\right) \delta \phi\right)\right\rangle+\frac{1}{2}\|\partial \delta \phi\|^{2}\right. \\
& +\frac{1}{2}\left\langle\delta \phi,\left(v+\alpha_{0}+\delta \alpha\right) \delta \phi\right\rangle+\left\langle\delta \phi, \delta \alpha \phi_{0}\right\rangle-\frac{1}{2 \lambda}\|\delta \alpha\|^{2} \\
& \left.\left.-\left\langle\delta \phi, f_{1}\right\rangle-\frac{1}{\lambda}\left\langle\delta \alpha, \alpha_{1}\right\rangle\right]\right\} .
\end{aligned}
$$

Keeping $\delta \psi$ fixed and solving the variational problem with respect to $\delta \phi, \delta \alpha$ we obtain the system of equations (3.20) for the generating set $\mathbb{B}_{j}$. We keep the 
same notations and assumptions as in Sect. 3, so this system has the unique solution $\delta \phi\left(\mathbb{B}_{j} ; \delta \psi, \phi_{0}, \alpha_{0}, f_{1}, \alpha_{1}\right), \delta \alpha\left(\mathbb{B}_{j} ; \delta \psi, \phi_{0}, \alpha_{0}, f_{1}, \alpha_{1}\right)$. Substituting this solution above we obtain a variational problem in $\delta \psi$, and its solution is, by definition, a function $\delta \psi\left(\mathbb{B}_{j}, \mathbb{B}_{k}^{\prime} ; \psi^{\prime}, \phi_{0}, \alpha_{0}, f_{1}, \alpha_{1}\right)$. Solving the full variational problem (4.41) we obtain a system of equations corresponding to (4.17), with some obvious changes connected with the form of the functional in (4.41). The equation for $\delta \psi$ is the same as the equation for $\psi$ in (4.17), and it has the unique solution given by (4.19), or (4.20), with $\phi$ replaced by $\delta \phi$. Substituting this solution, we obtain the system (3.20) for the generating set $\mathbb{B}_{k}^{\prime}$, which has the unique solution $\delta \phi\left(\mathbb{B}_{k}^{\prime} ; \psi^{\prime}, \phi_{0}, \alpha_{0}, f_{1}, \alpha_{1}\right), \delta \alpha\left(\mathbb{B}_{k}^{\prime} ; \psi^{\prime}, \phi_{0}, \alpha_{0}, f_{1}, \alpha_{1}\right)$. From this we obtain the representation

$$
\begin{aligned}
& \delta \psi\left(\mathbb{B}_{j}, \mathbb{B}_{k}^{\prime} ; \psi^{\prime}, \phi_{0}, \alpha_{0}, f_{1}, \alpha_{1}\right)=Q\left(\mathbb{B}_{j}\right) \delta \phi\left(\mathbb{B}_{k}^{\prime} ; \psi^{\prime}, \phi_{0}, \alpha_{0}, f_{1}, \alpha_{1}\right) \\
& \quad+\frac{1}{a\left(\mathbb{B}_{j}\right)} Q^{*}\left(\mathbb{B}_{k}^{\prime}, \mathbb{B}_{j}\right) a\left(\mathbb{B}_{k}^{\prime}\right)\left(\psi^{\prime}-Q\left(\mathbb{B}_{k}^{\prime}\right) \delta \phi\left(\mathbb{B}_{k}^{\prime} ; \psi^{\prime}, \phi_{0}, \alpha_{0}, f_{1}, \alpha_{1}\right)\right)
\end{aligned}
$$

which holds under the assumptions of Proposition 3.1. By this proposition the function $\delta \psi\left(\mathbb{B}_{j}, \mathbb{B}_{k}^{\prime}\right)$ can be bounded by $\left(2 K_{2}+1\right) \delta_{1}$, hence if $K_{2}\left(2 K_{2}+1\right) \delta_{1} \leqq c_{6}$, then it has values in the domain of the functions $\delta \phi\left(\mathbb{B}_{j}\right), \delta \alpha\left(\mathbb{B}_{j}\right)$, and we have the identities

$\delta \phi\left(\mathbb{B}_{j} ; \delta \psi\left(\mathbb{B}_{j}, \mathbb{B}_{k}^{\prime} ; \psi^{\prime}, \phi_{0}, \alpha_{0}, f_{1}, \alpha_{1}\right), \phi_{0}, \alpha_{0}, f_{1}, \alpha_{1}\right)=\delta \phi\left(\mathbb{B}_{k}^{\prime} ; \psi^{\prime}, \phi_{0}, \alpha_{0}, f_{1}, \alpha_{1}\right)$, $\left(L^{j} \eta\right)^{-2} \delta \alpha\left(\mathbb{B}_{J} ; \delta \psi\left(\mathbb{B}_{j}, \mathbb{B}_{k}^{\prime} ; \psi^{\prime}, \phi_{0}, \alpha_{0}, f_{1}, \alpha_{1}\right), \phi_{0}, \alpha_{0}, f_{1}, \alpha_{1}\right)=\delta \alpha\left(\mathbb{B}_{k}^{\prime} ; \psi^{\prime}, \phi_{0}, \alpha_{0}, f_{1}, \alpha_{1}\right)$.

They hold for the analytically extended functions on domains (3.21) with $\delta_{0} \leqq$ $c_{6}, K_{2}\left(2 K_{2}+1\right) \delta_{1} \leqq c_{6}$. We will use these identities in the future to solve various localization problems also.

\section{References}

1. Balaban, T.: Commun. Math. Phys. 167, 103-154 (1995)

2. Balaban, T.: Commun. Math. Phys. (a) 89, 571 (1983); (b) 96, 223 (1984) 